NISTIR 6850

\title{
Nanomanufacturing of Atom-Based Dimensional Standards
}

\author{
A Final Project Report of the \\ National Advanced Manufacturing Testbed
}

T.V. Vorburger, R.H. Allen, B. Damazo, J. Fu, D. Monk, M. Osti, J. Pellegrino, R. Rhorer, R. Russell, R. Silver, R. Sriram, K. Stouffer, D.A. Swyt, E.C. Teague, T. Wheatley, D. Wilmering

U.S. DEPARTMENT OF COMMERCE Technology Administration National Institute of Standards and Technology Precision Engineering Division Gaithersburg, MD 20899

January 2002

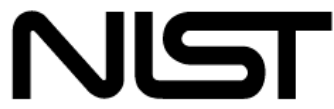




\title{
Nanomanufacturing of Atom-Based Dimensional Standards
}

\author{
A Final Project Report of the \\ National Advanced Manufacturing Testbed
}

\author{
T.V. Vorburger ${ }^{1}$, R.H. Allen ${ }^{2}$, B. Damazo ${ }^{3}$, J. Fu$^{1}$, \\ D. Monk ${ }^{4}$, M. Osti ${ }^{5}$, J. Pellegrino ${ }^{4}$, R. Rhorer ${ }^{5}$, R. Russell ${ }^{6}$, \\ R. Silver ${ }^{1}$, R. Sriram ${ }^{2}$, K. Stouffer ${ }^{6}$, D.A. Swyt ${ }^{1}$, \\ E.C. Teague ${ }^{1,3}$, T. Wheatley ${ }^{6}$, D. Wilmering ${ }^{5}$
}

\begin{abstract}
This report describes the accomplishments of the Nanomanufacturing of Atom-based Dimensional Standards Project, which operated as part of the National Advanced Manufacturing Testbed (NAMT), a program to research a wide range of leading edge technologies for manufacturing. As part of the NAMT vision, the Nanomanufacturing of Atom-based Dimensional Standards Project researched the techniques to manufacture and use dimensional standards at subnanometer levels of uncertainty. Its main area of application is the microelectronics and data storage industries where in the future, the critical dimensions of manufactured devices will grow continually smaller and where we anticipate that environments in which certain components are manufactured and used will become more specialized and exotic. This report discusses developments in atom-based step height standards, atom-based linewidth standards, a vacuum-based artifact transport system, a portable data logger, design and communication processes, and telerobotics.
\end{abstract}

Keywords: $\quad$ artifact transport; atom-based; collaborative design; data logger; dimensional standards; linewidth; nanomanufacturing; step height; telerobotics

\footnotetext{
${ }^{1}$ Precision Engineering Division, MEL

${ }^{2}$ Manufacturing Systems Integration Division, MEL

${ }^{3}$ Manufacturing Metrology Division, MEL

${ }^{4}$ Semiconductor Electronics Division, EEEL

${ }^{5}$ Fabrication Technology Division, MEL

${ }^{6}$ Intelligent Systems Division, MEL
} 


\section{Table of Contents}

1. Introduction 4

2. Basic Concepts of NAMT 4

2.1 The NAMT Program and Projects 4

2.2 The Overall Themes of NAMT 5

2.3 The NAMT Nanomanufacturing of Atom-based 6

Dimensional Standards Project and NAMT Themes

3. Description of the Nanomanufacturing of Atom-based 6

Dimensional Standards Project

$\begin{array}{lll}3.1 & \text { Motivation for the Project } & 6\end{array}$

3.2 Atom-based Artifacts 8

3.3 Results 9

3.4 Project Team and Partners 9

4. Project Accomplishments 10

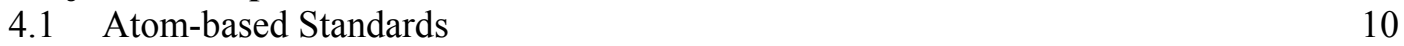

$\begin{array}{lll}4.1 .1 & \text { Goals } & 10\end{array}$

4.1.2 Step Height 11

$\begin{array}{ll}\text { 4.1.3 Linewidth } & 15\end{array}$

4.2 Artifact Transport System 21

4.2.1 Current Design and Results 21

4.2.2 A Second ATS Concept 23

$\begin{array}{lll}4.2 .3 & \text { Observations } & 25\end{array}$

4.3 Artifact Transport System Data Logger 26

$\begin{array}{lll}\text { 4.3.1 Introduction } & 26\end{array}$

4.3.2 System Design Overview 26

$\begin{array}{ll}\text { 4.3.3 System Design Specifics } & 27\end{array}$

$\begin{array}{ll}\text { 4.3.4 Internet Access } & 30\end{array}$

4.3.5 Results 30

4.4 Enhanced Design and Communication Processes 32

$\begin{array}{lll}\text { 4.4.1 Computer Visualizations } & 32\end{array}$

4.4.2 Collaborative Design (Engineering Tools) 36

$\begin{array}{lll}4.4 .3 & \text { Design Repository } & 38\end{array}$

4.5 Telerobotics 39

4.5.1 Control and Image Standards $\quad 40$

4.5.2 Remote Diagnostics Testbed 40

4.5.3 Recommendations 41

5. Conclusions and Future Work 42

6. Acknowledgments 42

$\begin{array}{lll}\text { 7. References } & 43\end{array}$ 


\section{Introduction}

This report describes the accomplishments of the Nanomanufacturing of Atom-based Dimensional Standards Project, which operated as part of the National Advanced Manufacturing Testbed (NAMT) in the Manufacturing Engineering Laboratory (MEL) at the National Institute of Standards and Technology (NIST). Formally operating from fiscal year (FY) 1996 to FY 2000, the NAMT was an ambitious program to research a wide range of leading edge technologies for manufacturing. As part of the NAMT vision, the Nanomanufacturing of Atombased Dimensional Standards Project researched the techniques to manufacture and use dimensional standards at subnanometer levels of uncertainty. Its main area of application is the microelectronics and data storage industries where in the future, the critical dimensions of manufactured devices will grow continually smaller and where we anticipate that environments in which certain components are manufactured and used will become more specialized and exotic. We pursue these ideas further in Section 2 of this report as we describe the basic concepts of NAMT. In Section 3, we provide a description of the Nanomanufacturing of Atombased Dimensional Standards Project itself. In Section 4, we give a final report of the accomplishments of the project, and in Section 5, we discuss future directions in research arising from this project.

\section{Basic Concepts of NAMT}

\subsection{The NAMT Program and Projects}

To support the development of solutions to the standards and metrology issues of modern information technology (IT)-based manufacturing, the NIST MEL has carried out a research and development (R\&D) program called the National Advanced Manufacturing Testbed (NAMT) [1-3]. The NAMT was developed to be a showcase for the future of manufacturing, demonstrating how machines, software and people can be networked together to achieve interoperability at all levels of a manufacturing enterprise. One feature of the NAMT is a stateof-the-art computer/conferencing laboratory [1]. At this facility, scientists and engineers from industry, NIST, academia, and other government agencies work together on measurement and standards issues in information-based manufacturing and on test methods for industry that are part of NIST's mission.

The projects of the NAMT have been characterized by: (1) collaborative industrial partners, (2) leading edge technologies, (3) development and use of advanced measurement technologies, (4) development of standards for manufacturing applications, (5) use of IT, and (6) tasks and processes at multiple sites on-line. The results of the NAMT are metrology techniques, interface standards, and infrastructural technologies and standards.

The NAMT program developed components of an information infrastructure for manufacturing, extending the capabilities of advanced computing, communications, and control technologies to multiple manufacturing applications and domains. The program also leveraged pools of manufacturing resources, including physical facilities, equipment, expertise, and software. 
The NAMT program began in FY96 with four five-year projects:

- $\quad$ The Nanomanufacturing of Atom-Based Dimensional Standards project, led by the Precision Engineering Division (MEL), focused on the distributed design, fabrication, and use of nanometer-scale dimensional artifacts.

- $\quad$ The Framework for Discrete Part Manufacturing project, led by the Manufacturing Systems Integration Division (MEL), aimed at developing a consensus-based framework for the interoperability of distributed manufacturing systems.

- $\quad$ The Development of Machine Tool Performance Models and Machine Data Repositories project, led by the Manufacturing Metrology Division (MEL), created a virtual manufacturing and inspection model and data repository to reduce the need for physical prototyping.

- $\quad$ The Characterization, Remote Access, and Simulation of Hexapod Machines project, led by the Intelligent Systems Division (MEL), aimed at measuring and extending the capability limits of parallel machines, and developing and evaluating techniques for open architecture control interface standards.

Subsequently twelve additional projects were added to the NAMT program [3], and these projects were led technically by NIST laboratories other than MEL. The projects focused on real-time construction site metrology, telepresence microscopy and microanalysis, virtual die design for information-based metal forming, radioactive seeds, internet commerce, a virtual cybernetic building testbed, tele-calibration of gas flow meters, remote calibration of electrical standards, next generation internet and intelligent collaboration and visualization technologies, IT solutions for small and medium size manufacturing enterprises, interface standards for arc welding, and internet-based calibration for radiation processing industries. In varied ways, these projects exploited the capabilities of high performance networking and advanced computing.

\subsection{The Overall Themes of NAMT}

Within the context of IT-based manufacturing, the unifying technical focus of the NAMT projects was development and demonstration of distributed and virtual manufacturing.

- $\quad$ By distributed manufacturing, we mean the cooperation in the overall manufacturing process among functionally specialized facilities at a variety of geographically separated sites by means of modern computer and communications systems.

- $\quad$ By virtual manufacturing, we mean both:

- real manufacturing by virtual enterprises, which come together by means of computer communications to produce a product or aspect thereof; and 
- virtual manufacturing by means of comprehensive and realistically predictive simulation of designs, processes and resulting products by means of quantitative modeling and qualitative animation.

\subsection{The NAMT Nanomanufacturing of Atom-Based Dimensional Standards Project and NAMT Themes}

As with each of the other three MEL-led NAMT projects, the Nanomanufacturing of Atombased Dimensional Standards Project was designed to jointly support:

- the overall NAMT program objective of support to IT-based distributed and virtual manufacturing; and

- the Precision Engineering Division's lead mission objective to provide, realize, and disseminate the international standard of length through length-measurement research and services.

As a result, the project used or supported the technologies of:

- distributed fabrication and use of nanometer-scale dimensional artifacts,

- $\quad$ computer modeling and simulation of mechanical systems and components,

- $\quad$ remote tele-operation of scanned probe microscopes (SPMs),

- $\quad$ all linked by means of advanced computers and communications for high-speed video, voice, and data transmission among collaborators.

These topics are described below.

\section{Description of the Nanomanufacturing of Atom-based Dimensional Standards Project}

This section is divided into four parts: motivation, atom-based artifacts, principal results, and the project team and partners.

\subsection{Motivation for the Project}

Two major trends in industry were driving forces for this project. First is the ever decreasing dimensions and tolerances posed by the semiconductor and data-storage industries. The

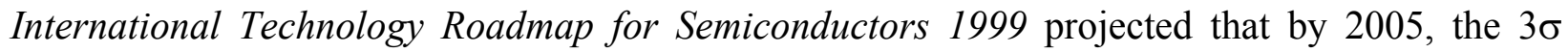
resolution requirement for metrology of circuit critical dimensions (CDs) will be as small as $1.2 \mathrm{~nm}$ [4], only a few silicon (Si) lattice constants. However, imperfections in linewidth standards limit the CD measurement accuracy. For example, the line from NIST Standard Reference Material (SRM) 473 [5], shown in Fig. 1a, is among the best calibration artifacts that industry and NIST can now produce in reasonable quantities. 
The imperfections in the line, its edge roughness and out of straightness, and irregular wall geometry, produce calibration uncertainties which are typically much greater than that of NIST's and industry's measurement instrumentation. This in turn results in limiting NIST's ability to meet the pressing needs of industry.

One of the project goals was to fabricate artifacts with feature geometries which are limited only

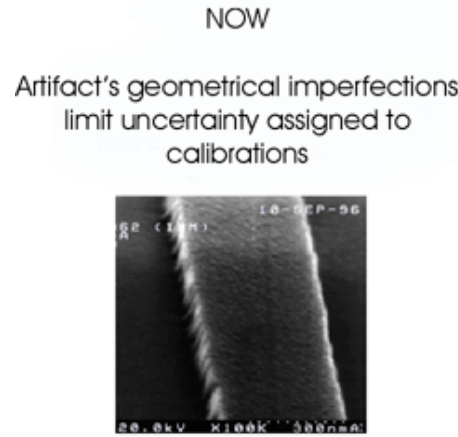

(a)

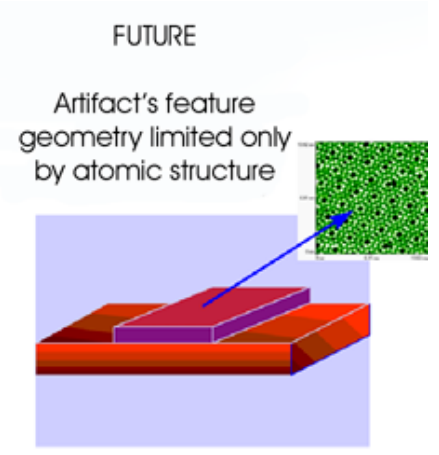

(b)

Figure 1 Properties of Linewidth Measurement Standards lattice constants, such as the one shown schematically in Fig. 1b. By collaborating with industry NIST hopes to develop such physical standards and to develop and put in place documentary standards to support their development. With these standards NIST will be in a much better position to meet the future needs of industry.

The second major trend in industry is the demand (resulting principally from the first trend) for ever cleaner and highly controlled manufacturing environments. These trends have been manifested in the move from clean rooms to mini-environments [6] and the use of standard mechanical interface, or SMIF, pods [7] for transferring materials between the manufacturing tools.

In advanced technologies such as extreme ultra-violet lithography [8], facilities for the various manufacturing and inspection steps may typically not be co-located with one another. The cost to bring expert staff, facilities, and materials to a common site is very expensive.

Based on this trend and the awareness that certain atom-based artifacts may live most of their lives in ultra-high vacuum (UHV) or at least highly controlled environments, Fig. 2 illustrates a vision of future distributed nanomanufacturing for an increasing number of specialized products such as x-ray photomasks [9] for advanced lithography which may require repair by a user in a separate laboratory from the manufacturing facility. This vision can be thought of as part of the larger vision of electronic (e)-manufacturing for semi-conductors [10] discussed elsewhere.

Figure 2 shows component fabrication, inspection, and control performed at three separate sites. Both the inspection and fabrication might take place in clean microenvironments. Such facilities might evolve further into UHV system environments with an artifact-transport system for transporting materials between the various manufacturing and inspection tools and geographical sites. 


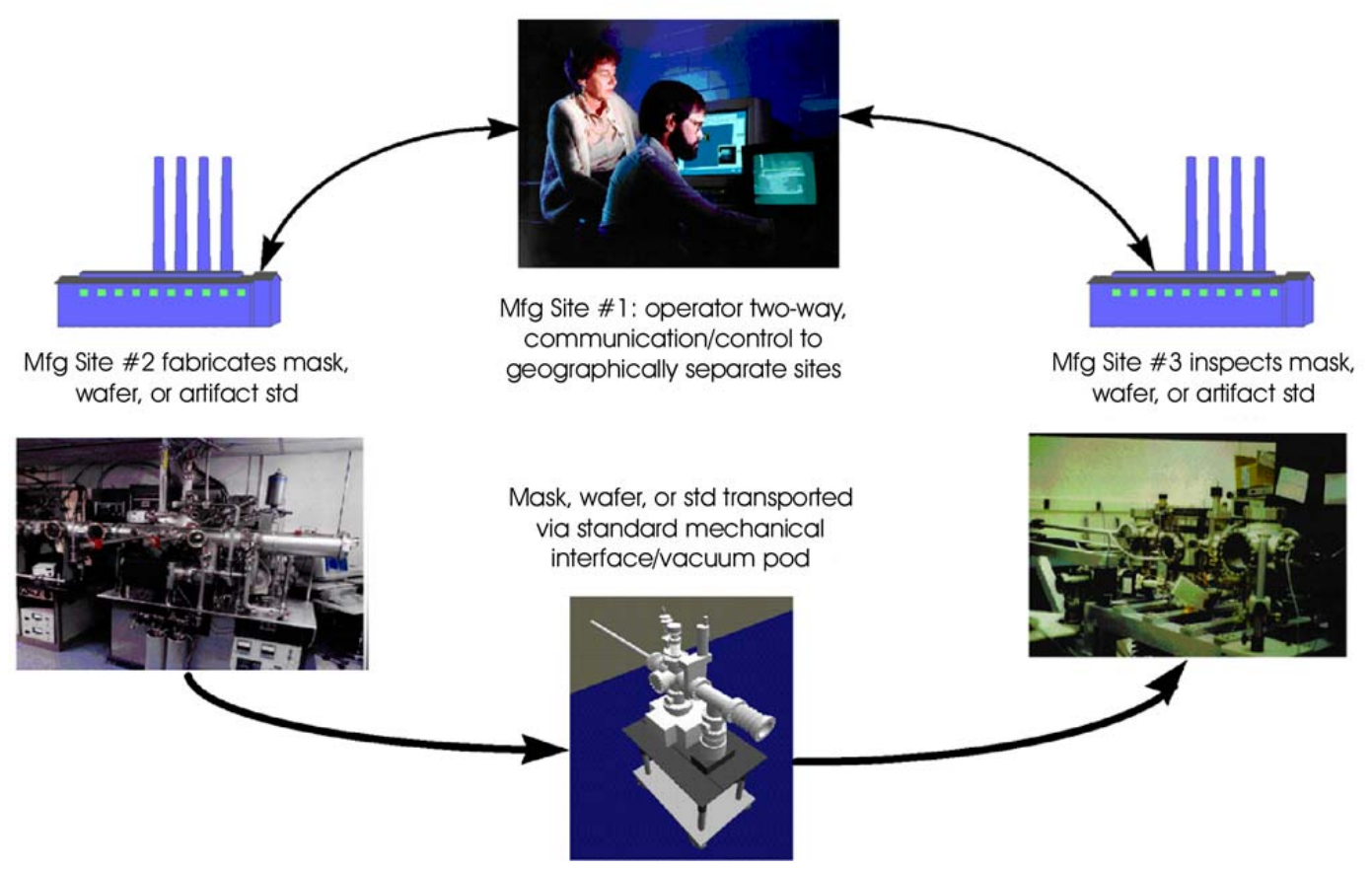

Figure 2 Vision of Future Distributed Nano-manufacturing in Microelectronics and Data-storage Industries.

The second major project goal was to develop a standard artifact-transport system along with standard mechanisms and artifact carriers for transferring artifacts between UHV systems. Such systems will enable the use of geographically-distributed facilities as shown in Fig. 2 and might require the development of a database dealing with the impact of various environmental conditions on the stability of atom-based feature geometry and dimensions. This database might also be needed for determining the final design of the artifact-transport system.

An additional activity of this project was to explore user and controller interfaces for telerobotic operation of scanned probe microscopes (SPMs), scanning electron microscopes (SEMs), and other scientific instrumentation that would be used for nanofabrication or inspection of atombased dimensional standards or devices. This is an area of great interest in the academic community for providing access to a remote scientific instrument and in the industrial community for remote diagnostics of instrumentation (e-diagnostics) [10] and remote operation of instrumentation across clean room boundaries. However, there are currently no documentary standards for operator or controller interfaces for most scientific instrumentation. It seems that the combined efforts of industry, government agencies and academia are needed to develop the technology and standards in this area.

\subsection{Atom-based Artifacts}

What do we mean by atom-based artifacts and how do we propose making them? The basic idea is to let the intrinsic atomic spacings of matter provide the scale for length related parameters of the artifacts. 
As shown in the scanning runneling microscope (STM) images of an Indium Antimonide (InSb) surface in Fig. 3, highly structured features on single crystal surfaces could serve as atom-based dimensional standards step height from the steps, roughness and line spacing from the stripe-like features, and linewidth as discussed before.

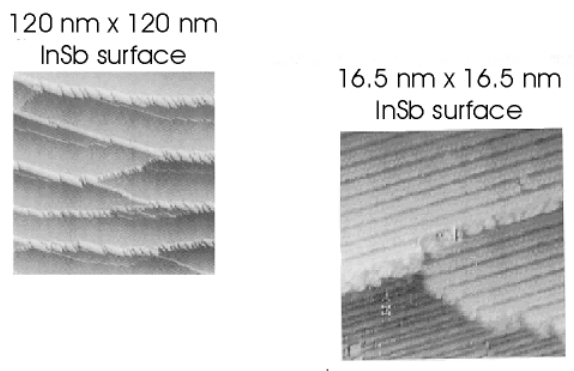

Figure 3 Concept of Atom-Based Artifacts

The current need is for feature sizes having linewidths on the scale of 10's to 100's of nanometers. For these features we must develop processes that do not result in excessively rough edges such as those shown in Fig. 3. The real challenge is then to make $100 \mathrm{~nm}$ scale features that result in straight and vertical edges and that are stable and transferable between NIST and industry.

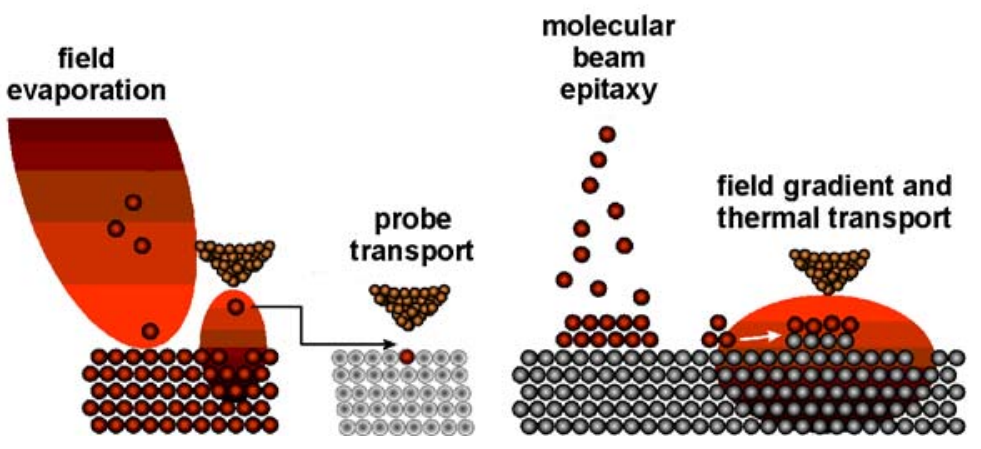

Figure 4 Nanomanufacturing Processes
Figure 4 shows some processes that may be used to produce atomicallydetermined features: electric-field evaporation, SPM transport of atomic or molecular species, molecular beam epitaxy (MBE), and thermal transport, perhaps with the application of electric-field gradients. In our initial efforts we have used MBE, field evaporation, and another process called selective dry etching.

\subsection{Results}

The results of the project include atom-based step height artifacts, prototypes for atom-based linewidth artifacts, the UHV artifact transfer system (ATS), a portable data logger for both real time and web-based monitoring of the ATS, and enhancements in design and simulation processes. Research questions still remaining include the need for standardizing mechanical interfaces, transfer mechanisms, and specimen carriers for UHV systems, and development of a standard controller and user interfaces for telerobotic operation of SPMs and other scientific instrumentation.

\subsection{Project Team and Partners}

The project team was composed of 16 people from the NIST MEL and the NIST Electronics and Electrical Engineering Laboratory (EEEL). In addition, colleagues at the University of 
Maryland, Thermo Microscopes, Texas Instruments, Veeco Metrology, and SEMATECH also contributed as partners.

\section{Project Accomplishments}

We now turn to accomplishments in the five principal areas of the project.

\subsection{Atom-Based Standards}

\subsubsection{Goals}

This component of the project had as its main goal:

- To develop and fabricate standards for step height and linewidth, whose dimensions and geometries are inherent in their structure and are tied to fundamental properties of matter, such as material lattice constants or atomic dimensions. The uncertainty arising from non-uniformity in the properties of such specimens should be significantly smaller than the uncertainties in instruments measuring them. Therefore, the uncertainties in their measured properties will not be limited by the specimens themselves.

Two other important goals follow from the one above:

- To develop specimen structures that may be exchanged between NIST and its collaborators. This implies either that the specimens themselves are robust and stable or that they are housed and moved in environmental support systems that avoid degradation of the specimens' dimensional properties. The environmental capability is important. The requirements of U.S. industry are such that NIST measurements may be required to demonstrate traceability of an artifact. If the structures are not sufficiently robust for them to be exchanged between NIST as the source of calibration and an industrial concern as the receiver, then the traceability of the calibrated atom-based structures might be compromised.

- To work with industrial collaborators in developing appropriate documentary standards to support efficient use of such artifact standards. This activity proceeds naturally from the successful development of the specimens themselves, but is not a trivial task. Standard algorithms for achieving closure between measurements taken by different laboratories have become more difficult to define as the amount of data generated by measurement systems has increased, owing to the increased speed of computers.

These goals proceed from the observation that the uncertainty that limits many types of precision measurements arises from the imperfection of the calibration artifacts that often serve as the sources of calibration for those measurements. When a highly accurate calibration system is used to measure a transfer artifact that is then used to calibrate another measurement system, the imperfection in the artifact itself often limits the accuracy of the 
calibration. On the other hand, some precision measurements rely on artifacts whose dimensional properties are based on the fundamental structure of condensed matter itself, either on its symmetry properties or its lattice spacings. A crystal lattice spacing is a quantity that is both small and precise. Smallness is important because the artifact can then serve as the source of calibration for other small quantities that require measurement; precision is important because it enables these calibrations to be done at high relative accuracy. The precision arises from the basic invariability of the properties of the crystal lattice itself.

The following discusses how such artifacts could be developed for precision step height and linewidth calibration.

\subsubsection{Step Height}

Precise specimens for calibrating step height are produced commercially $^{*}$ using optical lithography in Si. These standards are available with heights ranging down to $8 \mathrm{~nm}$. The uncertainty for these specimens ranges from about $0.5 \%(k=2)$ for steps on the order of $1 \mu \mathrm{m}$ to about $7.5 \%$ for an $8 \mathrm{~nm}$ step. An important source of the uncertainty for small steps is the uniformity of the step itself as a function of position on its surface, which in turn depends on the surface roughness of the bare $\mathrm{Si}$ and on the uniformity of the

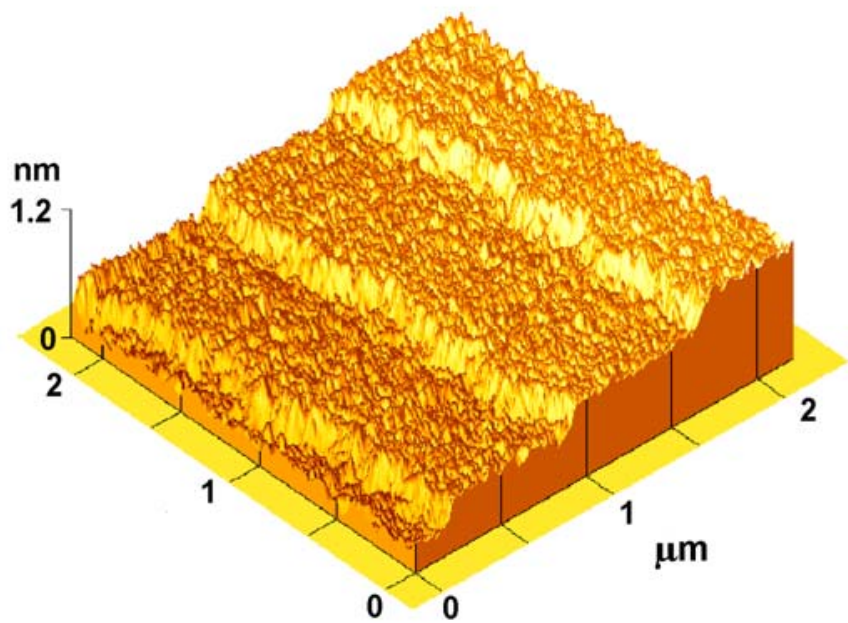

Figure 5 Image of $3 \mathrm{Si}$ Steps Obtained with the C-AFM. oxide growth process. This uniformity could be improved significantly with a step fabrication process under which the fabricated step height is determined by the lattice constant of the material from which it is made. If, in addition, the surface roughness is at the atomic level, then a value for the step height can be obtained with an uncertainty that is less than $0.1 \mathrm{~nm}(k=2)$. Williams et al. and others have shown that single atom step heights may be obtained on the (111) face of a Si single crystal by heating the specimen in vacuum [11]. The step height structure remains on the surface even after the Si specimen has been removed from the vacuum and the native oxide has formed. An image of three of these steps measured with NIST's calibrated atomic force microscope (C-AFM) is shown in Fig. 5. In principle, the step height is strictly related

\footnotetext{
* Certain commercial equipment, instruments, or materials are identified in this paper to specify adequately the experimental procedure. Such identification does not imply recommendation or endorsement by the National Institute of Standards and Technology, nor does it imply that the materials or equipment identified are necessarily the best available for the purpose.
} 
to the lattice constant of $\mathrm{Si}$ [12] and is equal to 313.560 137(9) pm, where the "(9)" indicates standard uncertainty in the last decimal place. Such an artifact can be used to calibrate a precise measuring instrument that is then used to measure another surface or another standard.

During the present project, we fabricated a number of $\mathrm{Si}(111)$ single-atom step height standards through a collaboration with the Materials Research Science and Engineering Center at the University of Maryland and later in a UHV system at NIST.

We then independently measured the value of the step height by using the Calibrated Atomic Force Microscope (C-AFM) $[\mathbf{1 3 , 1 4}]^{* * *}$, whose displacements in all three axes of motion are calibrated in terms of the wavelength of a helium neon $(\mathrm{HeNe})$ laser. The value obtained for the $\mathrm{Si}(111)$ step height using the C-AFM is $304 \pm 4 \mathrm{pm}(k=1)$, which gives us two independent values of the step height value differing by only $10 \mathrm{pm}$ or $3.2 \%$, thus imparting a higher integrity to the calibration artifact.

A third value of $312 \mathrm{pm} \pm 7 \mathrm{pm}(k=1)$ has been obtained from low energy electron diffraction [15] of the stepped $\operatorname{Si}(111)$ surface. A fourth value of $340 \mathrm{pm} \pm 30 \mathrm{pm}(k=1)$ has been obtained from grazing x-ray diffraction [16] of the stepped $\mathrm{Si}(111)$ surface. Using these independent results, we estimate a recommended value of the $\mathrm{Si}(111)$ step for use as a physical step height standard. The value predicted from the bulk lattice constant is many times more precise than the other values. If we were to weight the results as $1 / u_{c}^{2}$, where $u_{c}$ is the standard uncertainty for each, the weighted value would be determined entirely by the bulk lattice value and would have an extremely small calculated uncertainty. In our opinion, this is unrealistic. The value obtained from the bulk lattice constant may not represent the actual surface step height. By contrast, the other three values were obtained from measurements actually made on the surface of $\operatorname{Si}(111)$.

We obtain a recommended value for the step height using an approach based on methods explored by Kacker [14]. The first step is to develop a model of the probability distribution for the value of the step height. We take $313.56 \mathrm{pm}$ as the most probable value of the step height. Hence, the model distribution is peaked at $313.56 \mathrm{pm}$. In the absence of detailed knowledge of the distribution, we represent the state of knowledge by an asymmetric triangular distribution. To determine the lower endpoint of the triangle, we take the C-AFM result and subtract the expanded uncertainty $(k=2)$ from it. This leads to a value of $296 \mathrm{pm}$. To determine the upper endpoint, we take the low energy electron diffraction (LEED) result and add the expanded uncertainty $(k=2)$ to it. This leads to a value of $326 \mathrm{pm}$. We have thus interpreted the C-AFM result as indicating negligible probability that the true value lies below $296 \mathrm{pm}$, and the LEED result as indicating negligible probability that the true value lies above $326 \mathrm{pm}$. This is the basis for the selection of $296 \mathrm{pm}$ and $326 \mathrm{pm}$ as endpoints of the distribution. Because of its large uncertainty, the grazing x-ray result did not figure into our analysis. Using this model distribution, we obtain a mean and standard deviation of $312 \mathrm{pm}$ and $6 \mathrm{pm}$, respectively. This

\footnotetext{
** The references shown in bold are publications of the Nanomanufacturing of Atom-Based Dimensional Standards Project.
} 
leads to an "accepted" value and expanded uncertainty of $312 \mathrm{pm} \pm 12 \mathrm{pm}(k=2)$. This represents a relative expanded uncertainty of about $4 \%$ in the "accepted" value.

In continuing work we formed a working group whose purpose is to evaluate the usefulness of these step height specimens as calibration standards. The participants provided measurements of the $\mathrm{Si}(111)$ specimens to enable an estimation of the reproducibility of the step height measurements. Those providing information and measurements are shown in Table I.

Table I: Participants in the Laboratory Comparison

\begin{tabular}{|l|l|}
\hline Participant & Organization \\
\hline Debra Cook & Veeco Metrology \\
Hal Edwards & Texas Instruments \\
Joseph Fu & NIST, Precision Engineering Division \\
Ralph Nyffenegger & Park Scientific, now TM Microscopes \\
Paul West & Topometrix, now TM Microscopes \\
\hline
\end{tabular}

Each participant received his/her own specimen to test. We then received and analyzed altogether 36 topographic images from the five participants. Each instrument was calibrated independently according to the operator's customary procedure. Therefore, the absolute values of the measured step height results are not as important here as the fractional reproducibility of the step height results for each instrument. 
Typical results are shown in Fig. 6. Each topographic image consisted of several steps, six in this illustration. A step height measurement was then taken in several small areas where the data look to the operator as being free from noise and other measurement errors. The average step height value for the image was then calculated from the set of positions and the average step height value and standard deviation were calculated for the several images from each participant. The algorithm for calculating the step height value was a single-sided straight line fit as shown in Fig. 7. The results are shown in Table II. The order of the participants shown in Table II is not correlated with that of Table I.

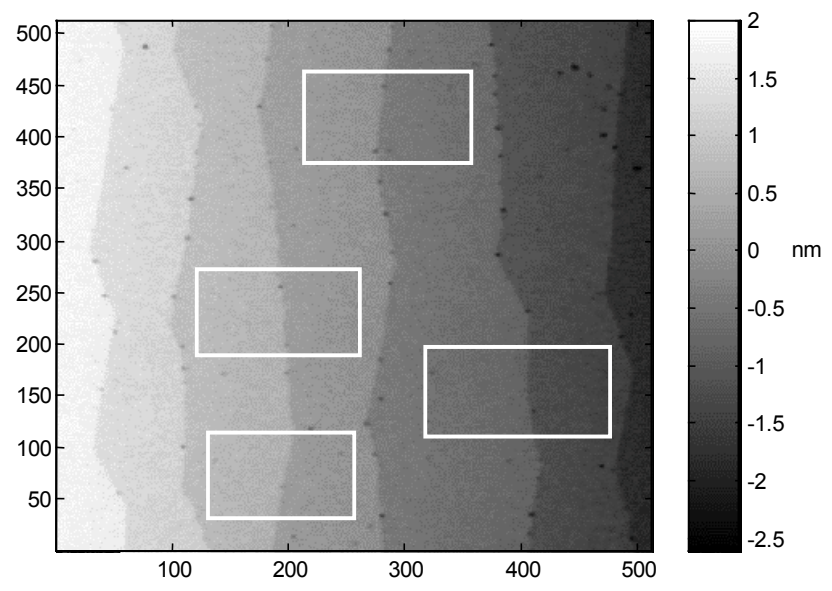

Figure 6 Topographic image of the stepped surface of $\mathrm{Si}(111)$ measured by one of the participants. The rectangles indicate typical areas used for determining the step height value.

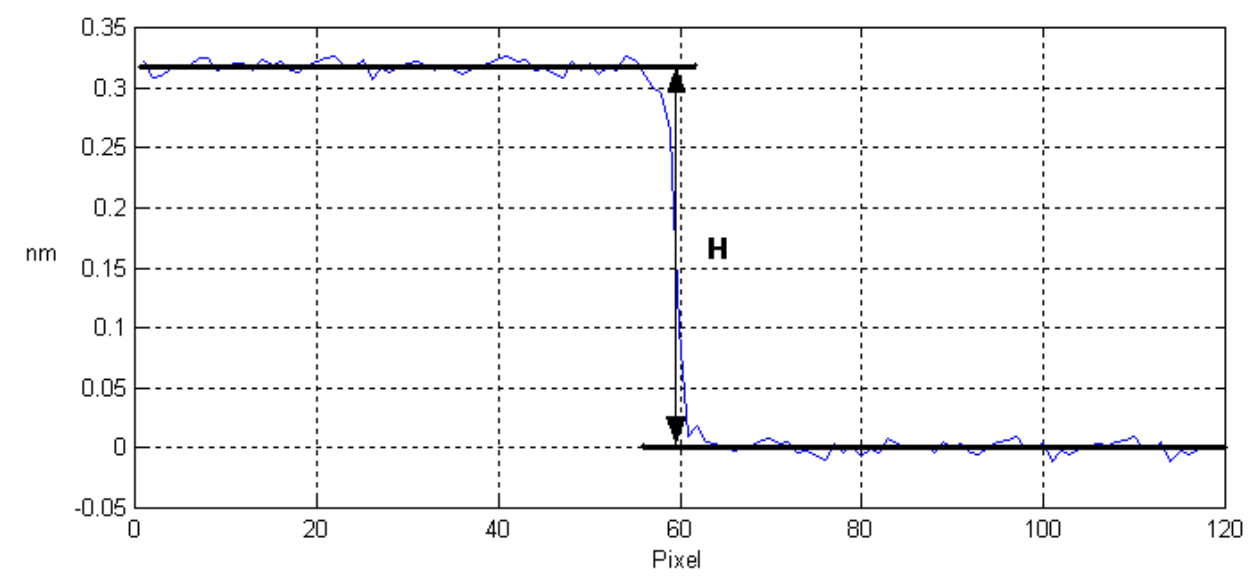

Figure 7 Illustration of the step height calculation for a single profile. 
Table II: Results for the Si Single-Atom Step Height Measurements. The order of participants here is not correlated with that of Table I.

\begin{tabular}{|c|c|c|c|c|c|}
\hline Participant & $\begin{array}{c}\text { Number of } \\
\text { Topographic } \\
\text { Images Used } \\
\left(\mathbf{N}_{\mathbf{I}}\right)\end{array}$ & $\begin{array}{c}\text { Average } \\
\text { Number of } \\
\text { Steps in } \\
\text { Each } \\
\text { Image }\end{array}$ & $\begin{array}{c}\text { Total Number of } \\
\text { Rectangular } \\
\text { Step Areas } \\
\text { Measured for } \\
\text { Each Participant }\end{array}$ & $\begin{array}{c}\text { Average } \\
\text { Step } \\
\text { Height } \\
\text { Value } \\
(\mathbf{p m})\end{array}$ & $\begin{array}{c}\text { One } \\
\text { Standard } \\
\text { Deviation } \\
\text { for the } \mathbf{N}_{\mathbf{I}} \\
\text { Values } \\
\text { (pm) }\end{array}$ \\
\hline $\mathrm{A}$ & 7 & 6 & 268 & 325 & 12 \\
\hline $\mathrm{B}$ & 9 & 4 & 90 & 302 & 8 \\
\hline $\mathrm{C}$ & 10 & 7 & 100 & 317 & 4 \\
\hline $\mathrm{D}$ & 4 & 6 & 180 & 306 & 3 \\
\hline
\end{tabular}

The results are very encouraging. The standard deviation for each set of measured images is $6 \mathrm{pm}$ on the average for results altogether. This suggests that with measurements from a single image of $\mathrm{Si}(111)$ step heights, one can obtain a calibration value reproducible to about $2 \%$ (one standard deviation $(1 \sigma))$.

A similar effort has taken place in Japan where a community of users of atomic force microscopes working under a committee of the Ultra Clean Society developed a standard procedure to use the Si single atom steps as a source of calibration for AFMs in the vertical direction $[17,18]$. Their results illustrate the variability of results that are possible with AFMs without such a standard. Their procedure uses a histogram analysis to determine the step height. The histogram analysis is more widely available in commercial software than the fitted straight line method. However, in our experience [19], the straight line method provides a more stable result.

\subsubsection{Linewidth}

Linewidth specimens serve as comparison standards for measurements of the smallest width features (critical dimensions, or CDs) on integrated circuits. Because the speed and density of integrated circuits improve as the size decreases, the CDs are manufactured to be as small as possible, thus challenging the capabilities for measuring them.

Linewidth measuring standards are currently available from NIST [5] and the National Physical Laboratory [20] in the U.K. for use as standards for optical instruments used to measure linewidth. However, the accuracy of the NIST specimens, and probably the U.K. specimens as well, is limited in large part by the quality of the edges on the lines themselves (see Fig. 1a). For example, the lines of the NIST specimens are produced with significant edge roughness leaving them with a variable width along the line length. In addition, the side-walls of these lines are not perpendicular to the substrate thus creating ambiguity as to where on the side-wall the lines should be calibrated and measured. The size of these effects imparts an uncertainty of approximately $40 \mathrm{~nm}(k=2)$ to a linewidth specimen with nominal width of $500 \mathrm{~nm}$. 
In this project, we explored the use of $\mathrm{Si}$ and Gallium Arsenide (GaAs) as counted atom linewidth standards [21]. These specimens would have linewidth features created by anisotropic etching such that the sides would be determined by the lattice planes of the crystal and would be perpendicular to the substrate. These lines would be heated in vacuum to remove the oxide, thus exposing the nearly perfect $\mathrm{Si}$ or GaAs lattice. The atoms in the lattice across the top width would be counted using a SPM or a transmission electron microscope (TEM) as the imaging system. The number of counted atoms across the top would then give us a measurement of the top width in terms of the lattice constant of silicon, which is known to a high accuracy. If the quality of the artifact can be preserved by maintaining it in vacuum with intermittent periods of gentle cleaning, it could be used as source of calibration for linewidth measurements made in UHV systems. It could also be used as a calibration artifact in atmospheric conditions at a slightly higher uncertainty.

\section{Atom Counting Methods}

Measuring linewidths accurately at very small feature dimensions is difficult as evidenced by the lack of industry standard artifacts. To analyze a line profile measured by an instrument requires advanced modeling methods to determine the linewidth because the chosen edge locations, upon which the linewidth value is based, depend on the response of the measurement system probe to the material. SEMs and optical microscopes have different responses to the edge of an etched feature. The SEM exhibits blooming and other edge related effects, and the optical microscopes show diffraction limited resolution. Both effects obscure the physical edge within the data. Currently the physical models for either type of reflection mode system are not sufficiently accurate for standard samples to be accurately calibrated and disseminated to industry. However, efforts are underway to develop sophisticated models. These efforts require a thorough evaluation and verification process. The development of models is advancing in parallel with efforts like ours to produce artifacts which are measured and characterized on the atomic scale, whose metric is then based on the crystal lattice. These atomically characterized samples can then be used in different ways to calibrate linewidth measurement tools as discussed next.

In the effort to develop a sample for accurate linewidth calibration, it has been proposed that a well defined linewidth structure whose dimensions could be measured with near to atomic accuracy would be useful. The structure or sample does not need to be the same as that measured in an industry measurement, on a product wafer for instance, if the calibration can be transferred to another artifact which can be measured in the industrial setting. On the other hand, products of some process levels are perhaps measurable atomically and are more directly transferable. At the root of both of these implementations is the idea of counting the atoms across a well defined structure and relating the number of atoms to the linewidth. This function requires a well defined sidewall or at least a well characterized sidewall close to vertical. The measurement uncertainty will be affected by variations in the sidewall geometry.

The sample we are developing, in part within this project, has therefore two primary capabilities

based on the atomic measurement concept [21]. First, the metric for the measurement can be 
based on the silicon crystal lattice. Second, the geometry of the edges can be measured or analyzed using the actual atoms that make up the edges.

Although both Si surfaces and GaAs surfaces form a native oxide, which is a few nanometer thick, the underlying crystal lattice has a well defined stable structure. The native oxide in $\mathrm{Si}$ is ordinarily disordered which is to say that the top 5 or 6 atomic layers of bound Si and oxygen, forming $\mathrm{SiO}_{2}$, are randomly ordered. Beneath this oxide there is a transition to a well ordered crystal whose orientation is dependent on the initial cut of the crystal; typically $200 \mathrm{~mm}$ wafers for semiconductor manufacturing have a (100) surface orientation. This oxide, however, maintains the topography of the underlying surface, as evidenced by the step and terrace structure persisting after exposure to atmosphere. A series of high temperature processing steps in UHV can remove the oxide and yield an atomically ordered surface. These surfaces at the crystal/vacuum interface, however, do not reflect necessarily the underlying crystal structure, but rather form a surface reconstruction more energetically favorable than the bulk crystal lattice because of the asymmetry of forces on the interfacial layer. The surface atoms have strong bonding forces on the crystal side and usually dangling bonds on the vacuum side, a condition that tends to restructure these surface atoms to relieve the stress. These Si reconstructions depend on the crystal orientation and processing, although there are several well established and understood reconstructions. An important fact is that the surface reconstructed atoms are commensurate with the underlying crystal lattice.

An outline of the overall process flow for atom counting in Si follows. The first steps are to take a Si wafer and etch an array of features using ordinary photolithographic techniques. Caution is necessary in removing excess photoresist and cleaning the etched wafer because contamination is a problem at the elevated process temperatures. Atomic surface reconstructions usually require the $\mathrm{Si}$ to be heated to $1250^{\circ} \mathrm{C}$ at a vacuum background pressure of less than $2.5 \times 10^{-7} \mathrm{~Pa}$ at temperature. Once the wafer is cleaned, it is diced and mounted on a quartz sample holder for loadlock into the UHV system. Currently, the die is cleaved rather than cut to avoid contamination from metals during the cutting process. The sample is then thermally processed and, after cooling to room temperature, transferred into the STM chamber. A feature is located and then measured with the STM. We navigate on the sample with piezo elements and a $200 \mathrm{X}$ telescope, which is capable of resolving the linewidth features of current interest. The STM, capable of lateral and vertical atomic resolution, then captures atomic resolution images across the linewidth feature which can be later analyzed with a variety of methods to count the number of atomic spacings across a feature. One method might utilize a template which is computer generated and laid over the image, or the atoms could be counted in a more pedestrian but automated fashion by counting the individual features.

Although we have just outlined the major steps in obtaining atomically resolvable silicon features, the process is not limited to $\mathrm{Si}$ and in fact GaAs offers some appealing alternatives, which address some of the problems associated with high temperature Si processing. Whether using Si or GaAs, underlying the entire process is the critical choice of crystalline orientation for the wafer as this affects the surface reconstructions and sidewall geometries.

Given the goal of fabricating lines in single-crystal silicon with countable atoms across the top surface, the choice of line geometry is constrained by the physics and chemistry of Si and 
existing etch technology and is dictated in part by the existence of certain energetically preferred stable faces of $\mathrm{Si}$. The $\mathrm{Si}(100)$ face is one example. Miscuts near (100) result in (100) terraces of varying widths separated by steps. The nonperiodic nature of the steps would complicate the counting of atoms across the surface. While perfect alignment is not possible, alignment close to a stable face results in larger terraces. Therefore, the first requirement is to orient the upper face of the line as near as possible to one of the stable faces of $\mathrm{Si}$.

Another factor is the expected arrangement of atoms on the upper surface of the line. The surface atoms reconstruct in a pattern commensurate with, but not identical to, the underlying bulk structure. The ideal candidate structure would have only a single possible reconstruction. Otherwise, if we encounter domains of various possible reconstructions, this increases the difficulty of the measurement. The well-known $\mathrm{Si}(100)-2 \times 1$ reconstruction has an advantage for atom-counting measurement because it is easier to resolve the resulting dimer rows (containing two atoms) from each other than it is to resolve atoms within a row. There are, thus, easy-count and difficult-count directions respectively perpendicular to and parallel to the dimer rows. The easy-count direction would be oriented across the line to be measured.

After selection of a crystal structure and its expected surface reconstruction, the samples are prepared and analyzed in the UHV environment. Subsequent to the UHV work, it is necessary to transfer the samples to other measurement systems. This commonly requires transfer of the artifact through air and therefore requires a stabilized surface, which will not compromise the dimensional integrity of the sample. One option is to simply allow the native oxide to form on the sample surface. Other options are the growth of oxide at elevated temperatures in varying pressures within the controlled environment of the UHV preparation facility. This may eventually result in more stable or smoother surfaces. We and others [11] have performed measurements on the $\mathrm{Si}(100)$ and (111) surfaces with the AFM in air after UHV preparation. The step and terrace structure with the subnanometer step heights remain clearly visible in the data for an extended period of time. These steps have been observed at least as long as six months after preparation, thus indicating that the topography remains stable enough for the atomic step heights to be observed with an AFM. This implies that, although there is no longer any observed lateral atomic order, there is enough quasi-order for the vertical steps to remain. This is important for using SEM analysis on previously measured, atomically ordered samples.

A problem for NIST and other national measurement institutes is finding a practical method for making traceable wafer linewidth measurements with uncertainties small enough to be useful in semiconductor applications. An atom-based linewidth standard might furnish part of the solution to this problem by taking an important place in the traceability chain between the definition of the meter and an optical or SEM measurement of a product specimen. We describe the following links in the chain:

1. Bulk Si lattice spacings are traced to the meter by measurements that combine $\mathrm{x}$-ray diffraction with optical interferometry.

2. Surface lattice spacings on the atomically ordered linewidth artifact can be determined from the bulk Si lattice spacings. 
3. An STM measurement of the artifact uses the lattice spacing to define an intrinsic "ruler" for distance measurements, and locates the edges of the line in terms of the atomic corrugation, thereby determining its width.

4. The artifact is passivated so it can be used in an ambient or near-ambient environment with a minimum change to its width. Two alternatives are also possible. First, a materialsindependent SPM measurement could be used to transfer the width from the atom-based primary standard to a stable secondary standard. Second, if required by the user, it may be feasible to transfer the specimen in its pristine condition under UHV to a second measurement system for an atom recount. Providing this capability is the subject of research described in Section 4.2.

5. Passivated or stable secondary standards are made available to manufacturers as SRMs. The manufacturer uses an SPM to compare the widths of the SRM and a representative product sample. The product sample thereby becomes an in-house standard with composition and geometry close to other product wafers.

6. Large numbers of measurements on product wafers are performed rapidly using optical or SEM intercomparison with the calibrated product wafer. Although the product wafer may not be stable for long periods of time, new ones can be made as required. The stable secondary standard would be used to determine the dimensional stability of the product standard, so a suitable schedule for generating new ones could be determined.

In general, each step in the above chain has an associated uncertainty which must be assessed in order for the final measurement to be traceable. For example, Step 2 is rendered easier by the fact that any surface reconstruction must be commensurate with the bulk, but lattice relaxation over a few atomic layers nearest the sidewalls would need to be taken into account. It is necessary to characterize any changes in width arising from the passivation at Step 4 . The use of product wafers at Step 6 minimizes differences between the unknown and the calibrated product standard during SEM and optical comparisons, but manufacturing process variations will cause residual differences between them to occur.

In related work, $\mathrm{Si}$ specimens have been produced and are being measured in an intercomparison of electrical, SPM, and SEM methods for linewidth measurement. The first set of experiments has produced excellent agreement [22]. Similar specimens will be prepared in UHV for measurements using the counted atom approach, a technique that in principle is limited by uncertainty components at the atomic level.

\section{Field Ion Field Emission Microscope}

When using the STM as a metrology tool to make measurements both on the atomic scale and on features with dimensions of a few hundred nanometers, detailed information about the tip shape is needed. In much of the previous STM work where the tip shape was taken into consideration, the tip shape was usually idealized as conical, spherical, or parabolic. The size of the tip can be accurately measured, as described below, with a field ion microscope (FIM). In previous work, 
the shape was most commonly modeled with a spherical end form, which gives good agreement for blunt tips. The tip radius can then be calculated based on this assumption.

The field ion field emission microscope (FIFEM) is an instrument which allows atomic characterization and cleaning of STM tips. We use it to measure the geometry of our STM probes on an atomic scale. The FIM is located in an adjacent UHV chamber to our UHV STM, and tips can be moved through UHV to the FIM before or after their use in the STM. The FIM was first developed in the 1950's [23] and until the development of the STM in 1982, nearly all FIM work was performed on tips with radii of several hundred nanometers. In an FIM image of a blunt tip it is possible to identify many crystallographic planes, although in an FIM image of a sharp tip there are typically only a few planes visible. As a result, blunt tips can yield significant information when studying grain boundaries, various types of dislocations, field desorption, and adsorption of other atoms. FIM analysis was also carried out to characterize sharp tips [24] for use in STM. This work was primarily intended to elucidate the tip preparation requirements focusing on the end of the tip for obtaining atomic resolution using STM. A more complete understanding of the actual tip shape was not of immediate interest, even though the overall tip dimensions and shape were recognized as important for understanding the tip robustness.

The current instrument has two modes of imaging and tip preparation. In the FIM, an imaging gas is bled into the chamber at about $1.3 \times 10^{-3} \mathrm{~Pa}$ and the tip is cooled to near $77 \mathrm{~K}$. The gas is ionized at the tip and accelerated towards a set of channel plates which generate an image on a phosphor screen. This image is digitally captured with a high resolution CCD camera and stored for further analysis and record keeping on a personal computer (PC). These images allow determination of the tip dimension before and after use, which aids in determination of damage to the tip during STM imaging. Although the details of dimensional image analysis are presented elsewhere [25], the basic idea is that known crystal planes are observed and the number of lattice steps between each crystal plane enables the determination of tip radius. During this process, the tip is cleaned but potentially dulled as well if the imaging voltage is increased beyond the minimum voltage required. Not all the atoms on a crystal plane are observed; only those near the edges where the fields are high are observed because this is where the imaging gas is first ionized. The second mode of this tool is the field emission mode (FEM). In this mode, the imaging bias voltage is reversed and electrons are emitted from the sample surface and impinge the phosphor. This method does not give detailed information about the tip geometry, although it does allow a calibration of the imaging voltage and geometry to give an estimate of tip sharpness. The primary advantage of this method is that by reverse biasing the tip we can clean the tip and remove the native oxide without dulling the tip. Additionally the tip is not cooled, so it only takes moments to prepare and analyze a tip. After cleaning and a determination of the tip sharpness, if the tip is measured to be sharp enough for atomic imaging, it is then placed into the STM and used.

In applying this method, we have found that for very sharp tips the spherical approximation used to explain the FIM measurement does not represent the tip shape accurately. As part of the NAMT effort we have developed a more complex model of STM tips using more complex geometries, which more closely match the physical tip shapes [25]. 


\subsection{Artifact Transport System}

To make use of a counted atom linewidth specimen with atomic scale accuracy, it will be necessary to maintain the surface in its original state where the atoms on the surface are observable with an SPM. That implies that such a specimen must be held in UHV so that any growth of oxide or other adsorbate is minimized. However, in order to be useful as a transfer standard with atomic scale accuracy, the specimen must be transferable between measurement systems at geographically different sites. An artifact transport system (ATS) has been developed to perform such transfers in UHV. The goal here is a system that would preserve the integrity of the standard, thus allowing the counted atom approach to be used at more than one location. Any contamination or build-up on the standards could bias the measurement of the dimensions.

The scope of the ATS task was to design, build or acquire, and test all necessary equipment to allow atom-based standard artifacts to be transferred: (a) between artifact manufacturers and artifact measuring systems, and (b) between either manufacturing or measuring systems and final users of the artifacts. Degradation of artifacts during transport was, therefore, a primary concern to this project. The convenience, reliability, and cost of the transport systems were also concerns.

Different types of transfer systems with different levels of vacuum were considered. UHV transport devices are needed for the most delicate specimens, because even in the high vacuum range (about $10^{-4} \mathrm{~Pa}$ ) a monolayer of gas could be absorbed on the artifact surface in seconds. When attempting to establish dimensional standards with uncertainties in the nanometer range, the effect of one monolayer may be significant. We anticipate a need for transferring artifacts with systems that maintain UHV in the range of $10^{-7} \mathrm{~Pa}$ or better to prevent the formation of a monolayer of contamination during expected transfer times of about $30 \mathrm{~min}$. Such a system would require on-board pumping, such as an ion pump and metal-sealed flanges.

To accommodate these requirements, a UHV system was designed specifically to transport artifacts between the MBE System in the Technology Building at NIST and the STM facility in the basement of the Metrology Building at NIST. A vital part of the ATS is the internal mechanisms or manipulators that actually handle the artifact inside the vacuum chamber and move it between the ATS and host system. For UHV systems, these mechanisms had to be carefully designed to avoid affecting the vacuum quality.

\subsubsection{Current Design and Results}

We studied the means of transferring artifacts while maintaining a UHV environment around the artifact at all times. We developed the ATS, which provides one solution to this challenge. In the UHV range it is necessary to provide continuous pumping for the chamber carrying the artifact because even a perfect leak-tight system will not maintain this level for more than a few seconds. Therefore, the ATS contains an ion pump, an associated battery power supply, and all other equipment on a portable cart. Although we have colloquially called the ATS a "vacuum suitcase" it is certainly not something that an individual can pick up and carry around. The design (Fig. 8) is based around the use of standard $150 \mathrm{~mm}$ vacuum flanges for the main transfer chambers. This size allows the operator to reach inside while the system is open to air. 


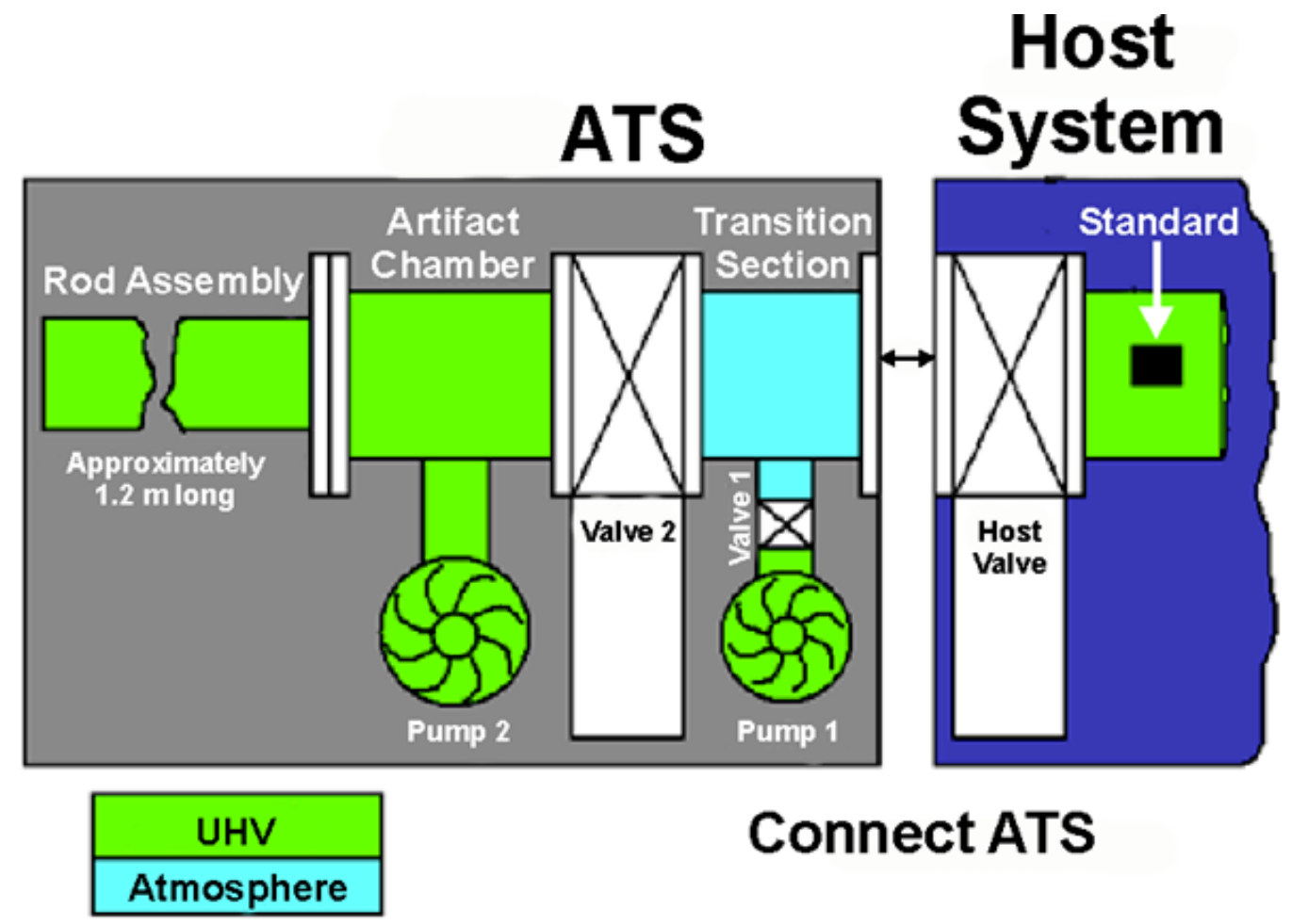

Figure 8 Schematic diagram of the artifact transport system (ATS) and the host system housing a standard sample ready to be transferred.

The ATS is conceptually similar to other types of transferring systems where an "air-lock" concept is used [26]. Before opening a valve or door into a host system the appropriate environment around that opening needs to be ensured. In the case of systems designed to prevent particulate contamination, such as SMIF [7] pods in the semiconductor manufacturing industry, an ultra-clean environment is maintained inside a module and only transfer devices of the same level of cleanliness can be attached for transferring parts. A similar concept has been used for the ATS. The transfer chamber is connected to the host chamber only when there is a UHV level pressure in the transition section of the ATS.

The ATS attaches to a port of a fabrication or diagnostic system (called the host system) by a standard conflat vacuum flange/seal. The host system must have a valve near this connecting flange. The ATS has a short pipe section (called the transition section) between the artifact chamber and the flange connection to the host system. There is a valve (Valve 2) between the artifact chamber and this transition region. The valve in the host system, and Valve 2 are not opened until the transition region is pumped to an adequate vacuum to ensure no significant increase in pressure in either the host or the artifact chamber. The artifact chamber is always maintained at UHV pressure.

After the vacuum joint is made, proper pressures are achieved, Valve 1 is closed, and Valve 2 and the Host Value opened, the sample holder (not shown) on the rod assembly is moved axially 
into position next to the sample. The sample holder then latches to the sample and is pulled back into the ATS specimen chamber. After the transfer is made, the valves can be closed and then the transition region of the ATS can be vented and the ATS can be disconnected from the host system. If the ATS artifact chamber has received the artifact from the host system, the ATS is then moved to a second host system. The vacuum flange is connected to the second host system, and the sequence of pumping and opening valves is repeated. The artifact can then be placed into the second host system with the manipulator. The artifact handling mechanism for our ATS was designed using standard vacuum feed-throughs and manipulators. Special hardware was designed and built to fit our particular artifacts and holders.

The ATS has successfully transferred an artifact from the UHV STM to the UHV MBE system about $300 \mathrm{~m}$ away. During transfer of the artifact from the UHV STM into the ATS chamber, the vacuum rose to approximately $3 \times 10^{-7} \mathrm{~Pa}$, then quickly settled to $9 \times 10^{-8} \mathrm{~Pa}$ and remained there as the ATS traveled to the MBE laboratory where the artifact was transferred into the MBE system. This demonstration verified that an artifact can be handled in such a manner that it never sees a pressure environment greater than $5 \times 10^{-7} \mathrm{~Pa}$ while being transferred between the two UHV systems. The pressure during a transfer was documented along with other parameters by a data logging system that was built as part of the PED-NAMT project. Details of this system are included in Section 4.3. A videotape of the transfer may be viewed on the internet [27].

\subsubsection{A Second ATS Concept}

A prototype lightweight and portable vacuum suitcase has been designed (Fig. 9) and partially constructed (Fig. 10). All components here were based on commercially available $70 \mathrm{~mm}$ UHV flanges rather than $150 \mathrm{~mm}$ flanges as in the original ATS. The system (Fig. 9) consists of two six-way crosses with a metal gate valve to divide two UHV compatible regions, an inner chamber and an outer chamber. Two ion pumps, $60 \ell / \mathrm{s}$ and $40 \ell / \mathrm{s}$, are used to pump the system. A portable power supply is capable of providing the power for the ion pumps and an ion gauge during transporting. 


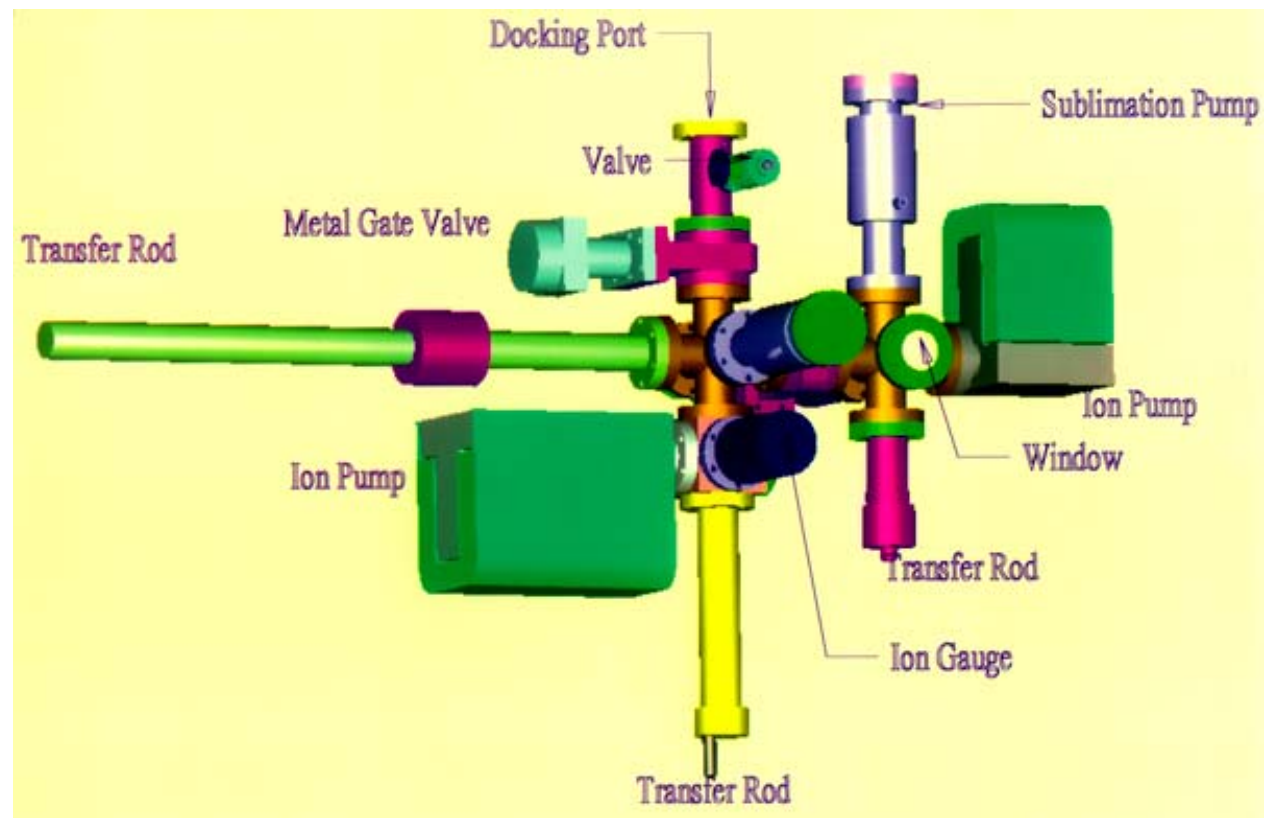

Figure 9 CADKEY diagram of a design for a small vacuum suitcase with $70 \mathrm{~mm}$ flanges.

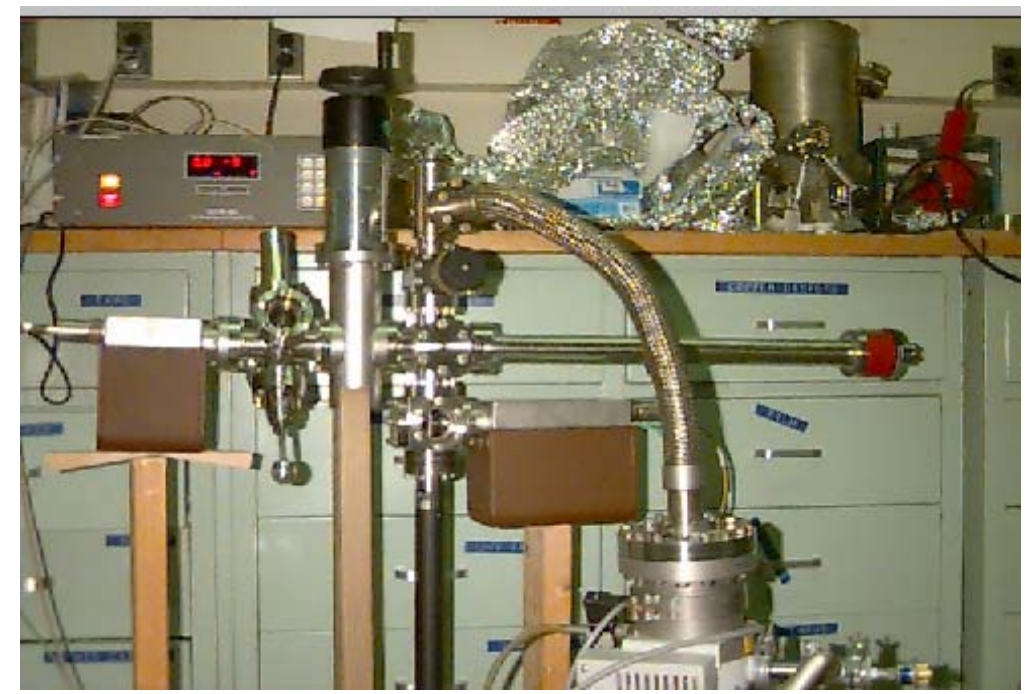

Figure 10 Photograph of partially constructed vacuum suitcase.

The novel design of the internal mechanism follows. A pair of transfer rods, mounted orthogonal to each other, allow transfer of the sample from stage to stage. The sample holder is shaped like a double yo-yo as shown in Fig. 11. The manipulators are two-pronged forks, which slide into different slots in the sample holder as a handoff is made. The design of Fig. 9 shows two stages of sample transfer in the suitcase itself. Due to the design of the sample holder, a fork-like grabber must also be installed in the host system prior to transferring the sample in or out of it. 


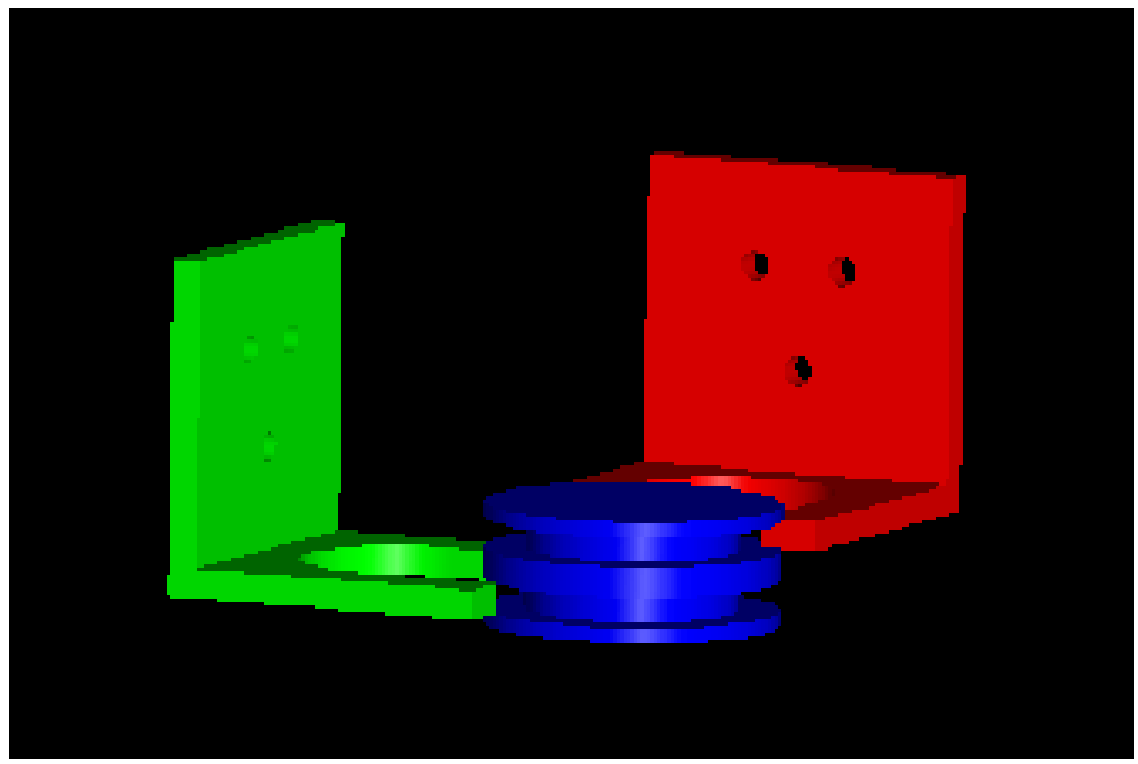

Figure 11 Sample holder for the small vacuum suitcase.

\subsubsection{Observations}

The basic concept of the ATS - producing an appropriate vacuum in a transition region prior to opening a host system - can be considered for other applications. One such future application may be handling wafers during part of the fabrication and inspection in the semiconductor industry. Such handling in a UHV environment is currently not being considered, but there may be a logical progression in some processes to better and cleaner environments. When a transfer of a standard or a production part is needed in the UHV range, the concept demonstrated by the ATS will be beneficial.

All the vacuum flanges and valves needed for this type of system are available as standard items from suppliers of vacuum equipment. The actual mechanisms for manipulating and handling the standards, or perhaps wafers in the future, need to be designed and built for particular situations. The handling mechanisms present challenges already encountered by robotic applications as well as the constraints of working in a UHV environment. These types of concerns have been addressed by others [28].

The NAMT project demonstrated the possibility of transferring a sample in the UHV environment between two locations. It is important to note that, whereas UHV chamber technology is standardized around conflat flanges, sample holders and manipulators are not standardized. Hence, for example, the $150 \mathrm{~mm}$ size ATS uses different sample manipulators than the $70 \mathrm{~mm}$ size. Before UHV transfers become widely used, the standardization of sample holders and manipulators will likely need to be addressed. The weight of the system is also an 
issue. If aluminum UHV hardware were used rather than stainless steel, a 50\% weight reduction would be possible.

\subsection{Artifact Transport System Data Logger}

\subsubsection{Introduction}

During the artifact transportation process, researchers are interested in the stability of the vacuum levels and the environmental conditions that may have affected the transport system (e.g., vibration levels, power failures, and equipment failures). A data logger has been developed and implemented to monitor the ATS, and in this section we discuss the system design and present results taken during the transport process described previously.

\subsubsection{System Design Overview}

The data logger uses an event-based algorithm to detect changes in the system and logs to the hard disk only these changes as a function of time. While this method of data acquisition continuously samples the system, it minimizes the amount of data stored and facilitates quicker analysis of data. One has to set a range over which the data may change without storing this as an event. The vacuum levels on the ATS are obtained using three different vacuum pumps (ion, sublimation and roughing), and the levels are read using two vacuum ionization gauges located in the artifact chamber (chamber one) and the transition section (chamber two). In addition, three accelerometers measure the real-time vibration levels seen by the chambers. The accelerometer levels are shown on real-time display charts on the data logger's front panel in Fig. 12. The $\mathrm{X}$ direction accelerometer measures positive values with forward motion of the ATS, the $\mathrm{Y}$ direction accelerometer measures side to side motion, and the $\mathrm{Z}$ direction accelerometer measures positive values in the upward vertical direction. 


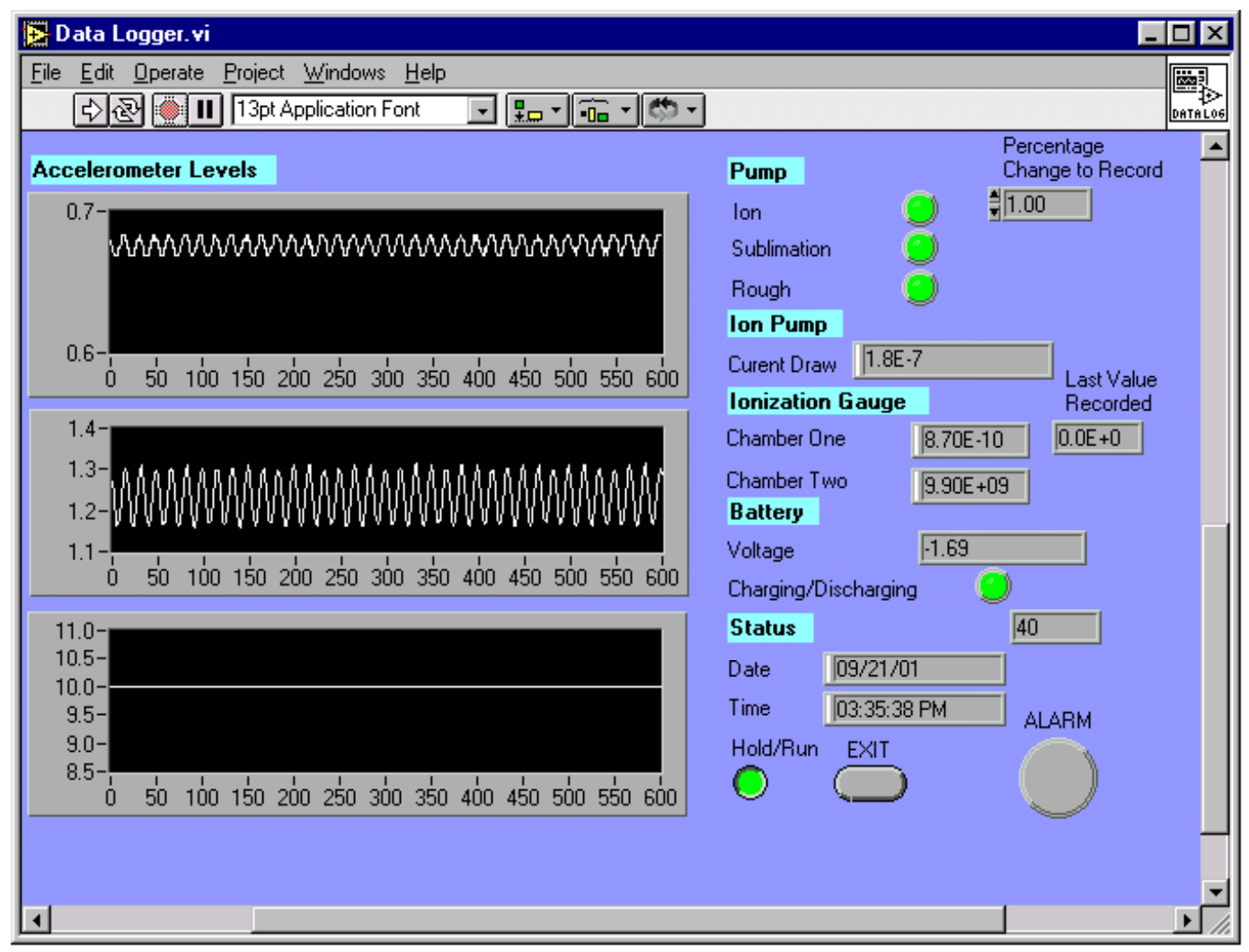

Figure 12 Data logger front panel is updated in real-time and is programmed in LabView. The X-direction accelerometer signal is at the top. The Z-direction accelerometer signal is at the bottom.

The data logger application software was developed in LabView on a laptop computer, which is mounted on the ATS with vibration isolation pads to minimize damage to the hard disk. In order to industrially harden the data logger, a PC104 format computer with a solid-state-disk (hard disk composed of solid-state memory) would be an alternative over the more delicate laptop used. In the event of a power failure, the laptop's batteries allow the data logger to continue operating for up to 1.5 hours.

A PCMCIA data acquisition card with 16-bit resolution and with a maximum $20 \mathrm{kHz}$ sampling rate is used to sample the analog signals. An eight channel sample-and-hold interface board connects the differential analog signals to the data acquisition card. Otherwise the data acquisition card samples the individual analog signals one after the other - not simultaneously. The ion pump controller and ionization gauge controller are interfaced to the laptop through the RS-232 communications port. The power for these instruments is from an uninterruptible power supply (battery powered).

\subsubsection{System Design Specifics}

Details related to the individual subsystems of the data logger are discussed in the following section. 


\section{Accelerometer Levels}

Three accelerometers are mounted on the ATS to measure the vibration (shock) seen during the transportation process. Three corresponding real-time charts show measured values in Fig. 12. Note that the third chart has a steady state value near 10 volts due to the gravity vector effect on the Z-axis accelerometer. The charts are set up for automatic vertical scaling and have a sampling rate of $1 \mathrm{kHz}$. The data acquisition card includes an oscilloscope that stores values to disk when a certain trigger level is exceeded. The values need to be post-processed to convert from the units of voltage to units of acceleration.

\section{Pump Status}

The three vacuum pumps draw their AC power from a custom made power strip. Mounted within the power strip are three current sensors that measure the current draw of the pumps. Three differential channels on the data acquistion card are used to read the current sensors. From the LabView front panel, indicator lights show red for no pump operation and green for pump operation as shown in Fig. 13. By setting the value for "Percentage Change to Record," the application will save to disk events due to changes in current draw of the pumps. In the event of equipment failure during the transport process, this information will help to determe if power was lost to the pumps, an individual pump failed, or the pump controller failed.

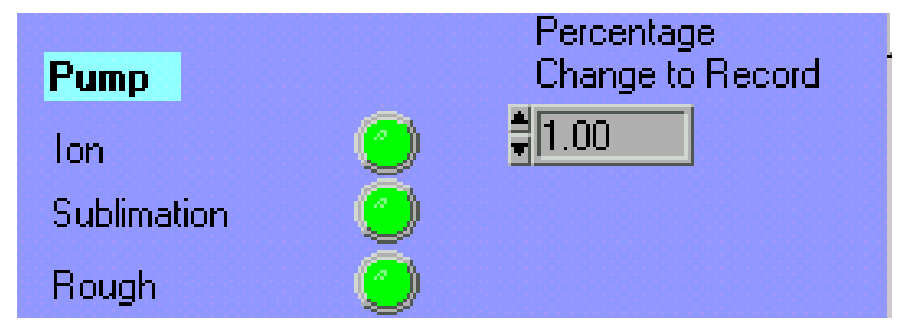

Figure 13 Vacuum pump status indicates the presence of a working pump.

\section{Ion Pump Current Draw and Ionization Gauge}

Both the ion pump controller and the ionization gauge controller are interfaced to the data logger via an RS-232 interface. Laptop computers typically only have one RS-232 port and in this application, an RS-232 switch was used to communicate with both controllers. The application software selects which controller to communicate with by sending control codes to the switching device. The current drawn by the ion pump controller is an indication of the vacuum level within the chamber and is shown in Fig. 14. This value is read continuously and stored to disk with a time stamp, when changes occur. The same is true for the ionization gauges. 


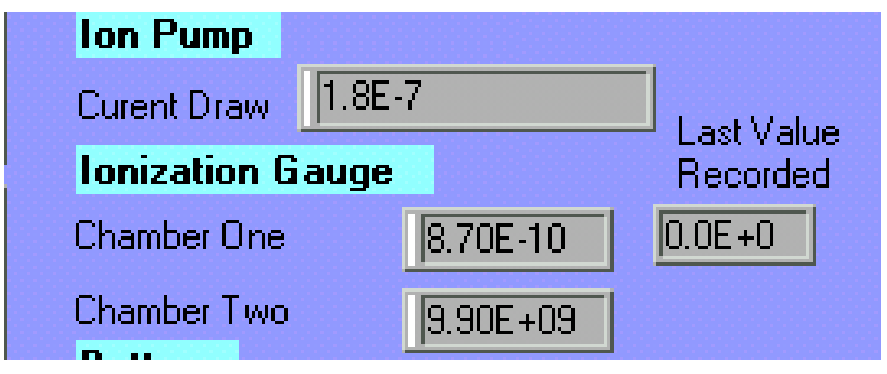

Figure 14 Current draw from the ion pump and ionization gauge values. For chamber two, the indicator shows that the ion gauge is turned off. The pressure indication in chamber one, shown here as $8.7 \times 10^{-10}$ Torr is approximately equal to $1.2 \times 10^{-7} \mathrm{~Pa}$.

\section{Battery Voltage Level}

During transport, the ATS is powered by an uninterruptible power supply, where the voltage of the battery is read using a channel on the data acquisition card. Changes in battery voltage are stored to disk with a time stamp and the voltage is displayed on the front panel as shown in Fig. 15. Based on a trend of increasing voltage (when unit is plugged into the wall) or decreasing voltage (during transport), the indicator represents a charging or discharging state of the battery. When the battery voltage drops below a threshold, an audible alarm sounds and the alarm indicator on the front panel lights.

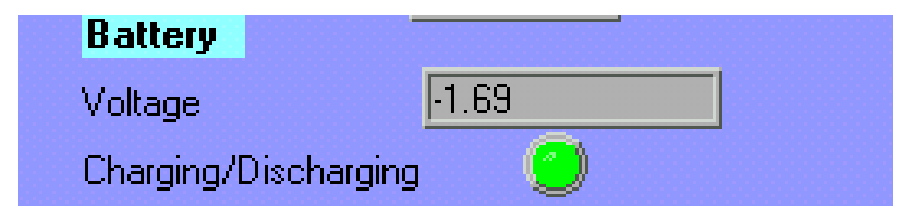

Figure 15 Battery status representation.

\section{Status and Execution}

Since an event-based algorithm is used to determine when to store changes in the physical system, a time stamp must also be stored. The status panel reads the laptop system clock and displays the information for the user. Internal to the LabView application is a high resolution counter programmable in ms that sets the sampling times of the accelerometers. In LabView, the start of an application is effected by clicking the run button. To stop or exit the data logger, the exit button shown in Fig. 16 is used. The indicator with the number "40" is used to determine how full the memory buffer is. Once the buffer is full, the contents are saved to disk. This indicator was only used in the software development process. 


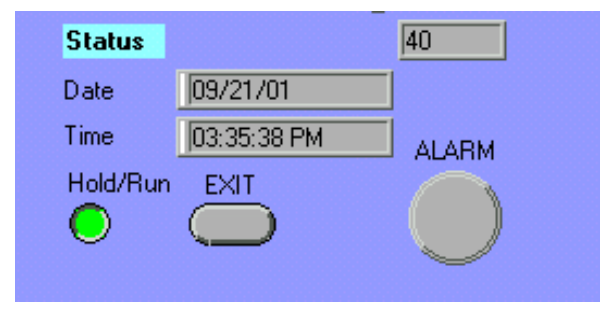

Figure 16 Status, controls and alarm representation.

\subsubsection{Internet Access}

This application includes the LabView 5.0 Internet Toolkit, which features the ability to view the data logger front panel status with an Internet browser. When the ATS was parked in the STM lab, the data logger was connected to the internal network and had its own internet protocol (IP) address. With the data logger running, snap shots of the front panel can be viewed and updated using the reload feature of a browser for a remote location. Also implemented is the ability to upload data files, collected during transport, to a server using a file transfer protocol (FTP).

\subsubsection{Results}

On July 27, 2000, a GaAs sample was moved into the ATS and transported from the UHV STM in the NIST Metrology Building to the MBE lab in the NIST Technology Building. The sample was successfully deposited in the MBE machine. The vacuum levels remained stable as shown in Fig. 17. The single spike in recorded vacuum level likely occurred when crossing over an expansion joint in the floor or moving into and out of an elevator.

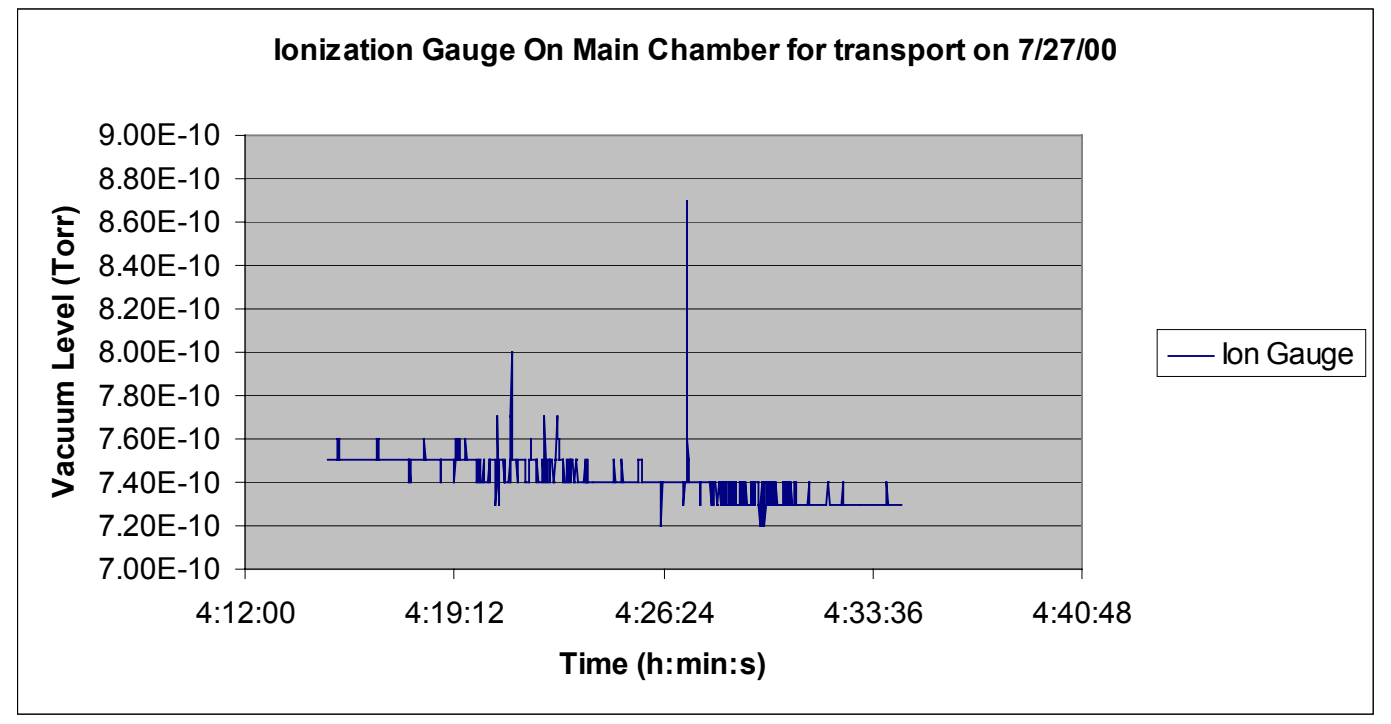

Figure 17 Ionization gauge plot showing trend in vacuum level during the transport. One Torr is approximately equal to $133 \mathrm{~Pa}$. 
During the transport, the ion pump current draw fluctuated as shown in Fig. 18. The current draw is proportional to the vacuum level and the average for the move was $0.12 \mu \mathrm{A}$, equivalent to a vacuum of about $2 \times 10^{-8} \mathrm{~Pa}$, with a standard deviation of $0.055 \mu \mathrm{amps}$.

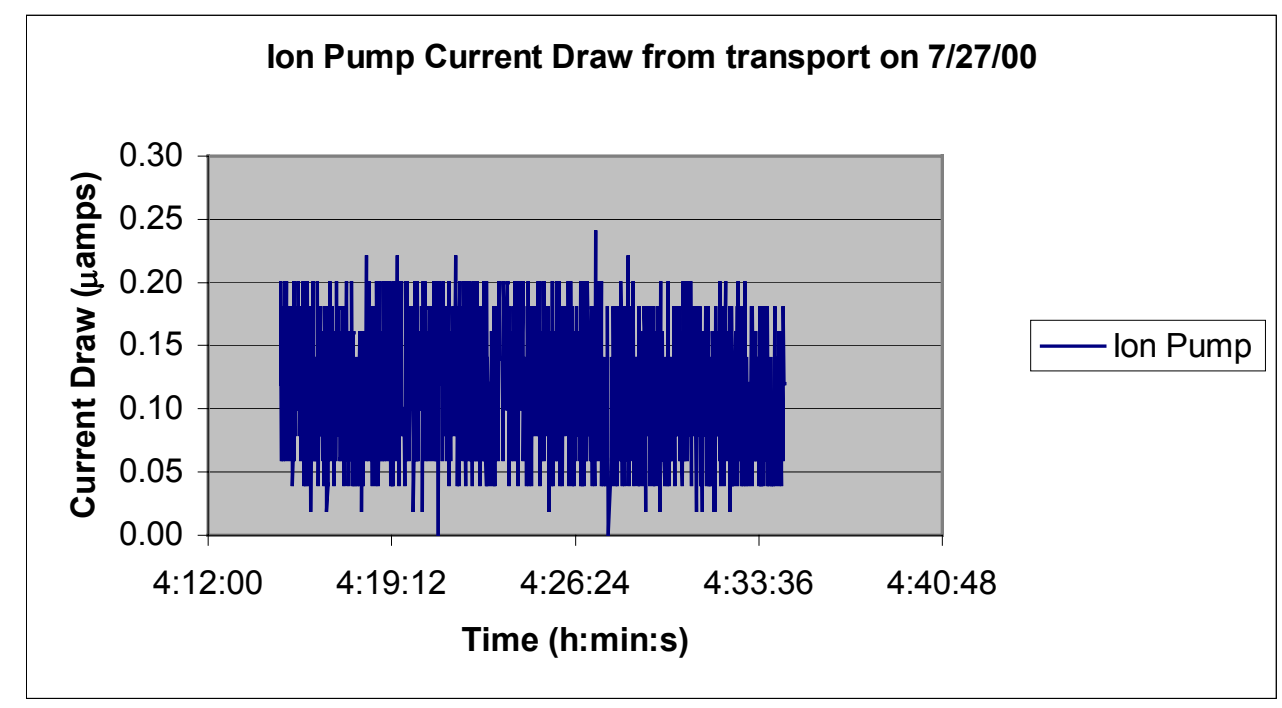

Figure 18 The ion pump current draw is an indication of vacuum level. The above data were taken during the move between buildings.

Figure 19 shows a plot of acceleration levels read from the X-and Y-axis accelerometers that were mounted on the ATS. The data represents the noise floor. Due to a malfunction, the Z-axis data were not available at this time.

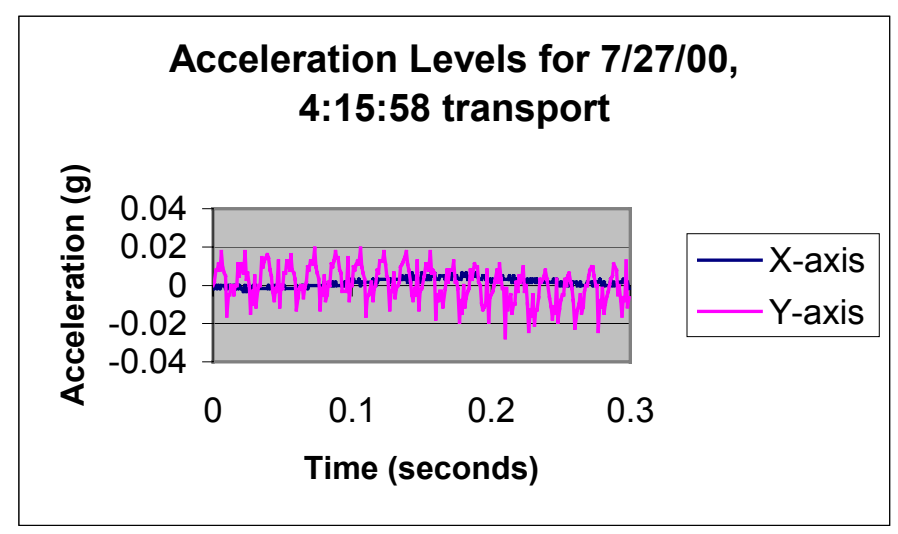

Figure 19 For the time period listed, the plot shows the acceleration levels for the $\mathrm{X}$ - and $\mathrm{Y}$-axis. This represents the noise floor. 
Overall, the data logger performed reliably and indicated stable operation of the ATS and excellent vacuum. New generation linewidth standards may need to be transported under UHV to insure that no contamination or exposure to air degrades them. The data logger will provide researchers with information on vacuum level stability and environmental conditions impacting the transport systems to study the effects on the standards.

\subsection{Enhanced Design and Communication Processes}

In this section, we discuss work to enhance the design and communication processes of the ATS. Specifically, we discuss how simulations were used to aid in visualizing function and assembly, how collaborative software tools were used to reduce design time and improve communication, and how the design and the basis for the design are encapsulated in a design repository. Further details of this work are given elsewhere [29-33].

\subsubsection{Computer Visualizations}

We performed four types of modeling and simulation tasks in support of this project: simulating the internal mechanism of the ATS, representing its overall geometry, simulating its assembly sequence, and modeling the STM tips used for probing surface geometry.

Simulating the internal mechanism of the ATS was important to describing and understanding the transfer procedure so that mishaps could be avoided during the transfer. The components of the internal mechanisms, which have distinct, precise linear motion and rotary motion, were designed in ProEngineer and I-DEAS. These are the mechanisms for moving specimens from the MBE system and placing them in the STM, or vice versa. Realistic simulation of the assembly was achieved by exporting the ProEngineer and I-DEAS models to Alias PowerAnimator - a flexible animation tool developed for SGI workstations - via the Initial Graphic Exchange Specification (IGES). Figures 20 and 21 show snapshots from the simulation.

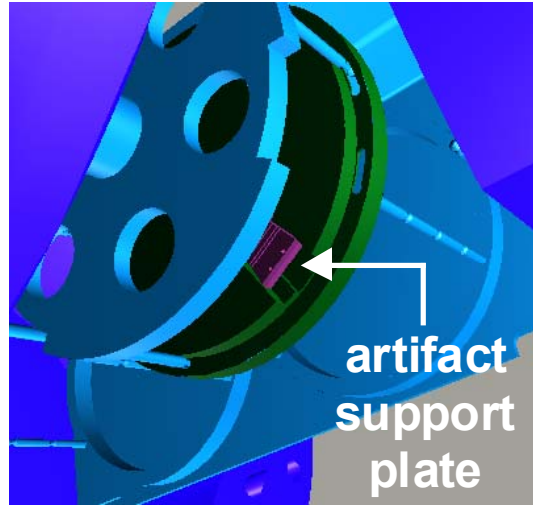

Figure 20 Picking the artifact from MBE system, before redesign

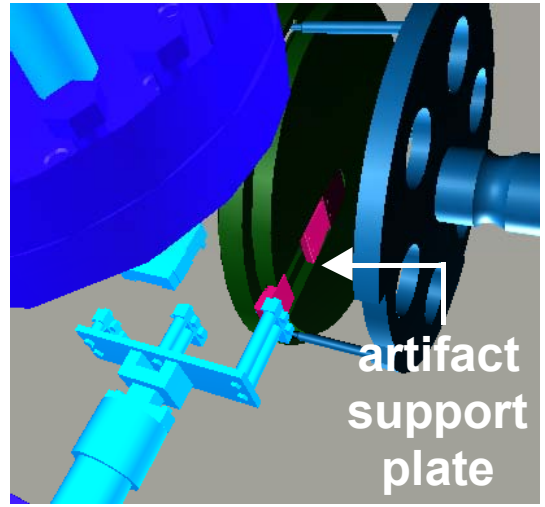

Figure 21 Placing the artifact in the STM, before redesign 
Because this simulation was developed on a computer workstation, this particular evaluation was conducted in a face-to-face meeting in NIST's NAMT Lab [29]. This laboratory was developed, in part, to enhance synchronous communications, such as meetings and conference calls. Part of that enhancement is a linked videowall, which can project images from any of the workstations in the lab (and other sites around NIST) via a high-performance network.

When the simulation was displayed in the NAMT Laboratory during the meeting, one physicist identified a problem with the mechanism. As depicted in Figs. 20 and 21, for example, the initial configuration had a support plate that could slip when placed in a vertical orientation, taking the artifact along with it. Since the same mechanism is being used to unload the artifact in STM, as shown in Fig. 21, the same slipping problem occurs. Along with other suggestions, this ultimately resulted in the engineers modifying the mechanism. This again was developed into a simulation that was reviewed and approved, and now is available as a demonstration model.

Representing the ATS geometry was our next task to enhancing collaboration. The assembled designs of the ATS were presented to the team members so that each could evaluate details of the design. In this effort, we chose an asynchronous approach so that the team members could perform their evaluations at different times and places. We chose Virtual Reality Modeling Language (VRML) and the Internet as communication media because they support our aim by allowing team members to view graphic files at their convenience.

We used CADKEY and ProEngineer converters to export the assembly models to VRML models. These models were made available through the Internet and thereby accessible to the entire team at one time. We then edited these VRML files to complete the ATS system assembly, which is shown in Fig. 22.

Because VRML objects can be manipulated without losing their representations as solid models, the final assembly still retained the computer aided design (CAD) model rendering to help engineers to visualize space constraints, such as whether the UHV system fits into the cart or not. VRML also provided the team members the feel of virtual reality, which means that one can obtain a sense of the VRML model using a walk-through of the simulation supported by VRML browsers. 


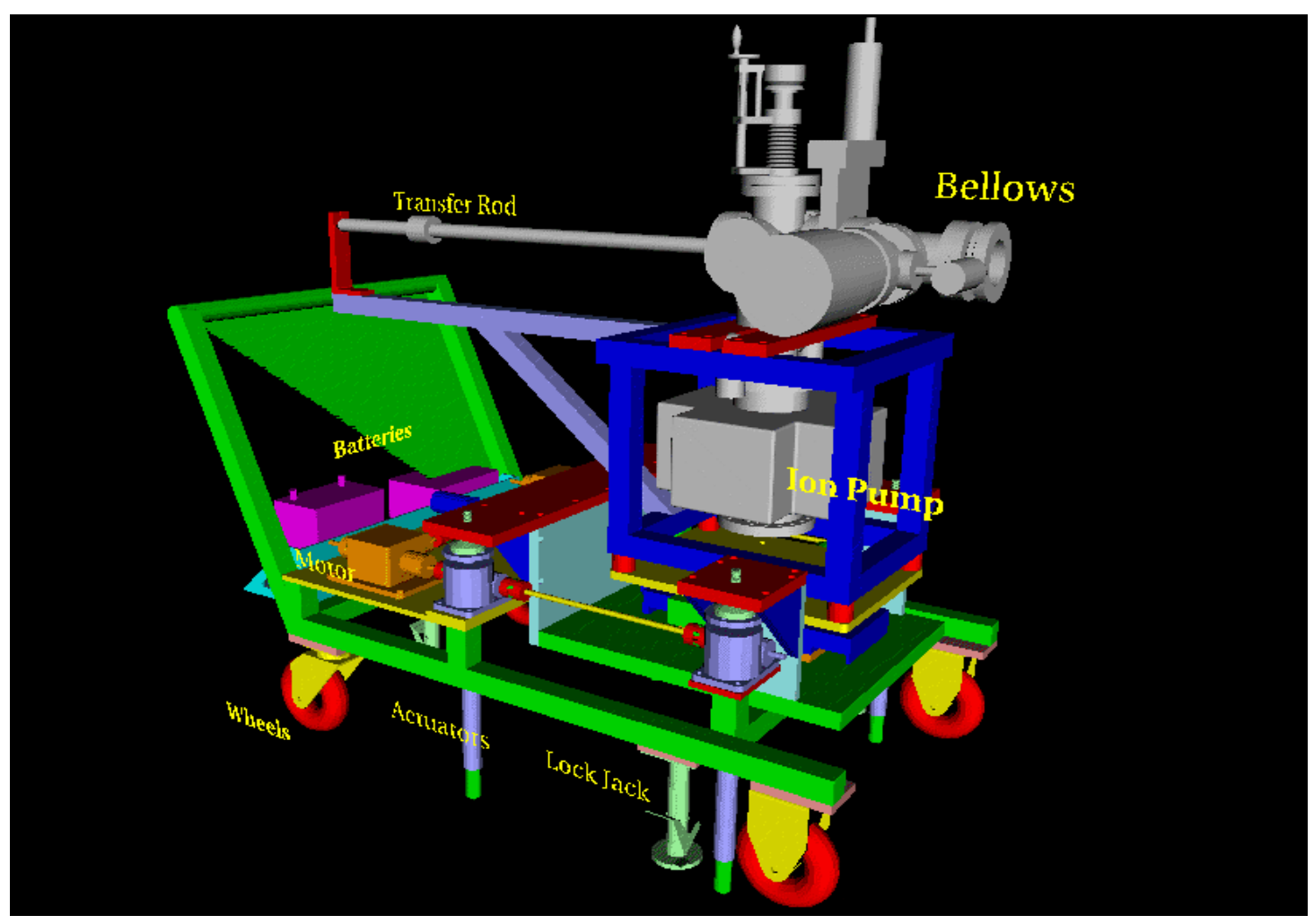

Figure 22 Assembly of cart and UHV system as a unified VRML model.

Simulating the assembly sequence for the ATS, our third effort, was aimed at improving the design process. Digital mock up of this assembly sequence was crucial to identifying problems of assembling components, and to saving time and effort by avoiding these problems during actual assembly. The engineers needed information on UHV system assembly procedures from the physicists.

Following the success of the second effort, we used VRML animation for asynchronous visualization of the assembly sequence. Similar to our earlier efforts, we used CADKEY converters to export the assembly models that the engineers developed to IGES models, which we imported into ProEngineer. We edited these VRML files to complete the cart and UHV system animation. Figure 23 shows a sample VRML snapshot of the simulation, an exploded view of the UHV system and the cart at the beginning of the animation.

Ultimately, the team used the animation sequence synchronously to agree on the optimal assembly sequence. This animation also proved quite useful in revealing problems that previously had gone unnoticed. 


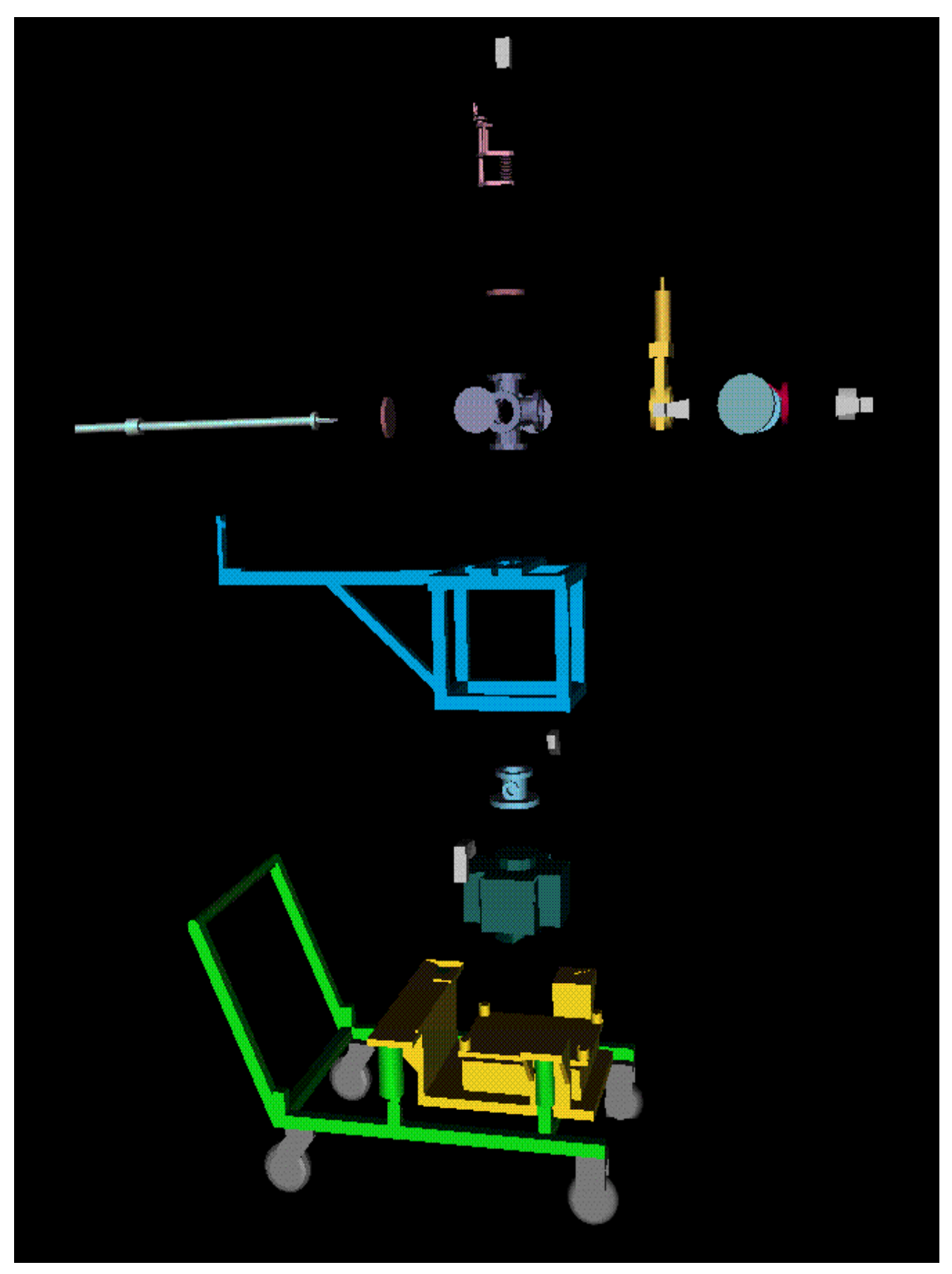

Figure 23 An exploded view of the ATS at the start of the assembly sequence.

Computer modeling of the FIM of STM tips enabled us to understand the structure and quality of the STM tips. Gray-scale images obtained from SPMs are functions of both the geometrical properties of the emitting tip and the electronic properties of the surface. Creating accurate models of SPM tips is important in the area of dimensional metrology because features such as lines and surface profiles obtained with an SPM, for the purpose of measuring linewidth, are strongly dependent on the tip geometry. The modeling of an SPM tip involves surface reconstruction of the atomic structure of the tip from a FIM image using image processing techniques. This modeling required both a reasonably good understanding of single crystal 
properties and crystallography, as well as the basic principles of operation of an FIM. Our results are discussed elsewhere [25]. An example of our results for a (111) Tungsten (W) tip is shown in Figure 24.

In future work, we intend to simulate the dynamic response characteristics of scanning probes. In all SPMs, the signal obtained is a strong function of the spacing between a mechanical tip and a test surface. In a STM, this signal is the quantum mechanical tunneling current, which is produced by a small electrical bias voltage between the tip and the surface. Here the tunneling current is an exponential function of the tip-surface spacing, such that a change of tip-surface spacing of one atomic diameter produces approximately a factor of ten change in the tunneling current. In an AFM, the signal is proportional to the net atomic force between atoms on the SPM tip and those nearby on the test surface.

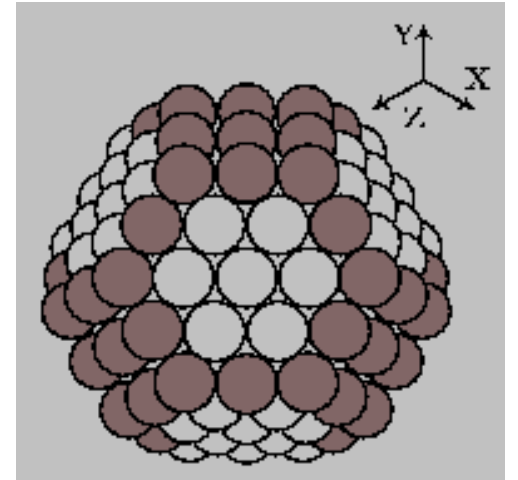

Figure 24 Atomic model of a W STM tip with (111) orientation.

Both types of microscopes and other similar microscopes produce a gray scale representation of the test surface topography by electromechanically servoing the tip to maintain a fixed spacing from the surface. By recording the servo-control signal as a function of the tip $(\mathrm{x}, \mathrm{y})$ position as it is scanned across the surface one may obtain a profile of the test surface. Repeated parallel scans are used to build up a gray-scale image. We plan to develop and demonstrate a model to simulate the response of the SPM to varying step heights or other sharp variations in surface topography. The response of the instrument to both external acoustic and/or seismic perturbations will also be carried out. We will also provide visualizations of the varying response characteristics of the SPM to changes in the resonance frequencies of the mechanical structure of the SPM, to varying bandwidths of the servo control loop, and to different values of noise in the servo control loop. Such modeling would be of great value to the SPM community and to SPM manufacturers.

Simulations of fabrication processes such as MBE, thermal and field evaporation, and STM pickup and transport of atoms and molecules may also be carried out. Here the challenge of obtaining adequate scientific models of the processes would be of greater interest than executing the simulations.

The use of web-enabled tools for modeling and simulation aids significantly in carrying out the above mentioned tasks.

\subsubsection{Collaborative Design (Engineering Tools)}

To share the different types of information associated with the ATS design among the team members, we implemented a collaborative workbench $(\mathrm{CW})$ that was designed specifically for platform-independent design and manufacturing collaboration. The $\mathrm{CW}$ consisted of two principal parts: an Internet-accessible portion and a platform-specific collaboration notebook. The Internet-accessible portion ran on a local server and consisted of a Project Area, containing 
project-specific information such as drawings, specifications, and schedules, and a Document Vault, which stored files of any type that could be uploaded via client World Wide Web browsers. The collaboration notebook is software local to each team member's workstation, and was primarily used as a whiteboard for pasting comments on existing documents and drawings.

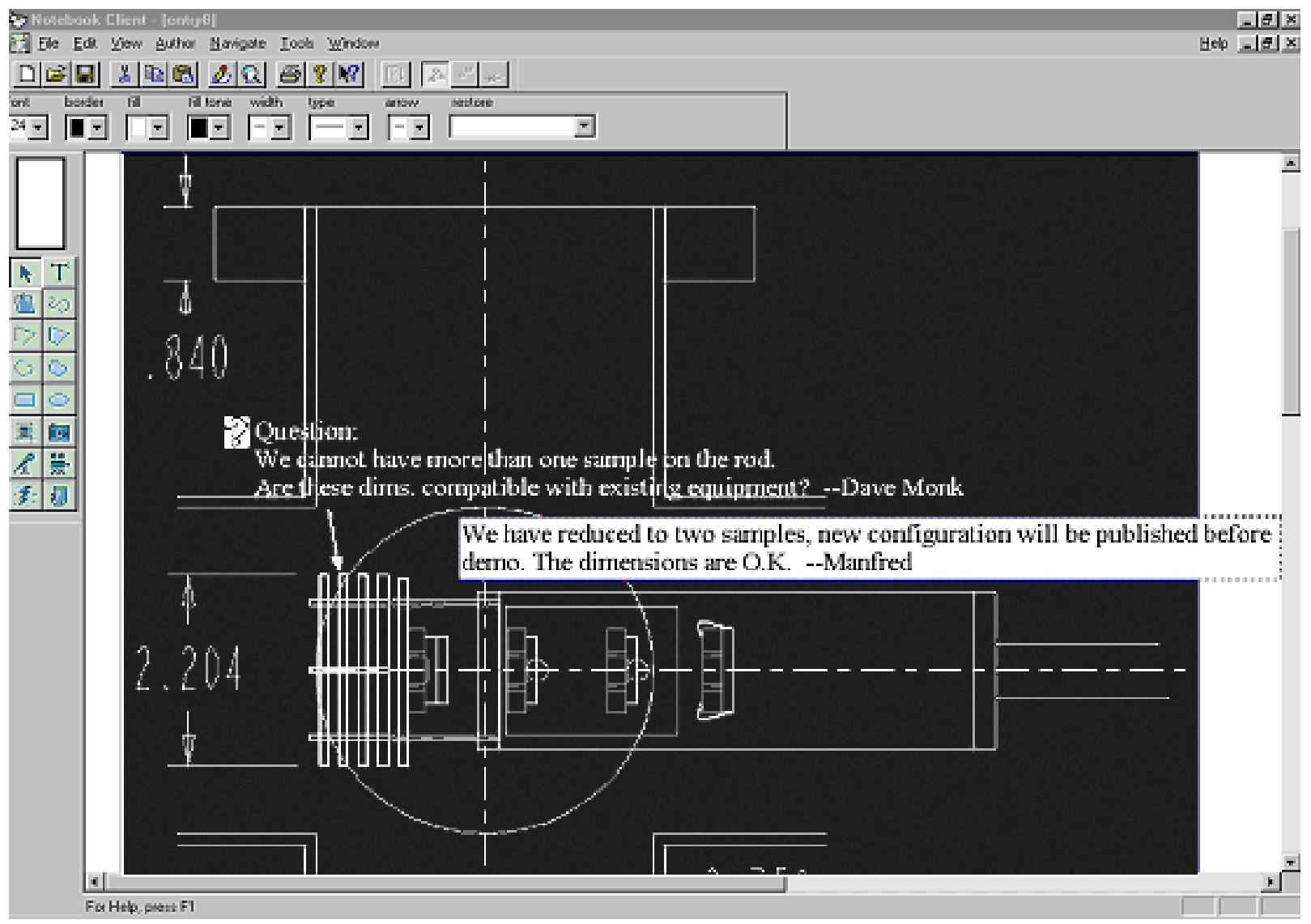

Figure 25 Using the collaboration notebook, this figure captures a sample asynchronous interaction between team members Dave Monk and Manfred Osti over the ATS internal mechanism shown in Figs. 20 and 21.

Based on the specifications for the ATS, designers created and represented ATS components and assemblies on their respective CAD systems. The designers published their designs on the CW and informed project team members of the newly available CAD drawings via automatic email. Team members then commented directly on the $\mathrm{CW}$ representations of the CAD drawings (example shown in Fig. 25), and those comments were republished with the drawings. This process continued until team members reached a consensus, or until face-to-face meetings helped resolve conflicting issues. Similar processes occurred with documents such as reports and schedules and with digitized photographs of manufactured components. Environments like the $\mathrm{CW}$ can be effective in helping teams overcome the problems associated with diverse computing environments and heterogeneous data formats, and they can be effective in facilitating 
consensus-based decisions among several collaborators. This technology improved the design and manufacturing processes for the ATS in specific instances.

\subsubsection{Design Repository}

In an effort to preserve the design documentation for the ATS, we encapsulated the form, function, behavior and rationale of each ATS component in a NIST-developed design repository (DR). The DR captures form, function and behavior of each component within a design and maps it onto the data elements such as objects, classes and relations. As an example, the pumping system takes ambient air inside the chamber and reduces it with a turbo pump to a high vacuum, which in turn gets pumped down with an ion pump to UHV. Schematically, we can represent the pump down system as a single artifact, having an input state characterized by ambient air, and an output state (destination) characterized by UHV. However, the functions of this system's two-fold vacuum pumping are intertwined. The turbo pump produces an intermediate, high vacuum in the ion pump chamber by taking air and decreasing pressure in a controlled way. The turbo pump is then valved off and the ion pump completes the process to achieve UHV in the chamber.

To populate the design repository, we represented engineering geometry, function, and associated behavior, as well as taxonomies of generic functions and flows of the transport system. We modeled geometry with digital photographs and VRML images of actual CAD drawings. We represented function with linked textual descriptions, and we captured behavior with mathematical expressions. Through an evolving user interface, shown in Fig. 26, this representation serves to capture the more than 50 parts and systems of the ATS in such a way that the information relating to form, function and behavior is accessible and browsable to subsequent designers and other interested parties. 


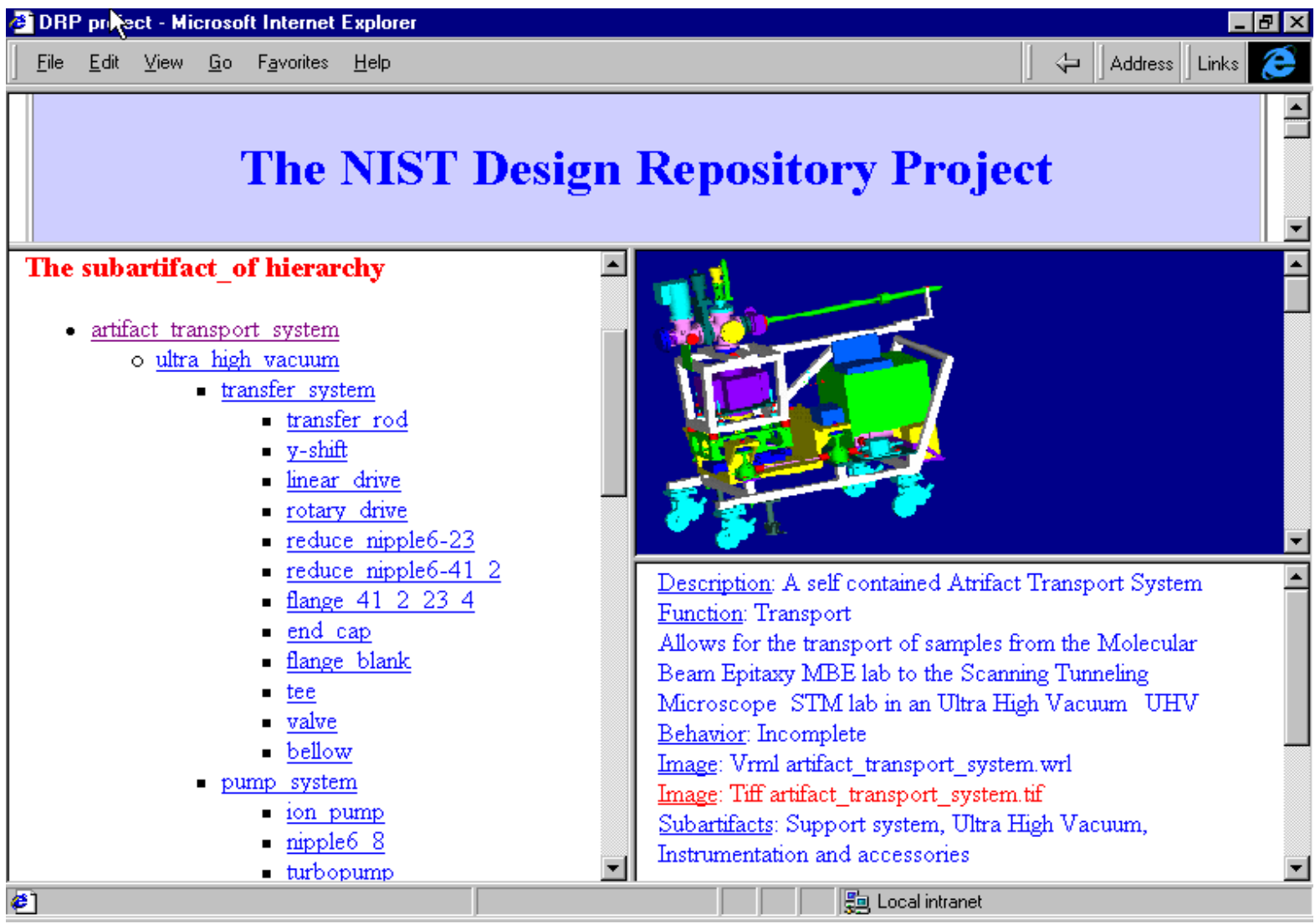

Figure 26 A sample user interface. The graphic screen depicts one VRML view of the overall system. The lower screen describes the system, its basic function and its subartifacts. The system hierarchy is defined on the left.

\subsection{Telerobotics}

In our vision of future microelectronics manufacture, a variety of micro-, nano-, and quantum-electronic devices with tightly controlled dimensional features could be fabricated under high vacuum in systems located in clean room environments at geographically distributed sites. Each site could specialize in one or more functions in the overall manufacture of the mask or device such as $\mathrm{R} \& \mathrm{D}$, design, fabrication, inspection, processing, or repair. Within this distributed nanomanufacturing system, partially processed devices, mask and device design data, and real-time diagnostic information need to be moved rapidly and efficiently among different geographical sites.

With the requirement for device fabrication in clean room environments increasing, there is a growing need for automated control of processes and robotic manipulation of wafers and carriers. Remote, "through the clean room wall," teleoperation of SPMs used for inspection of fabricated microstructures and remote "from a vendor's firm" teleoperation of such SPMs for instrument troubleshooting (e-diagnostics [10]) may become commonplace and necessary.

Traditional teleoperation usually involves a close physical and visual interaction with the mechanism, typically moving a single joint at a time to achieve the desired motion. It can 
become tedious, for repetitive motions such as scanning, as well as prone to error due to operator inattentiveness. Any robotic mechanism requires continual updates to its motors, even when stationary, due to the effects of gravity. This data is required to be deterministic, that is, arriving on a regular basis much like the individual frames of a movie, to ensure smooth operation. Lowlevel teleoperation over long distances can be difficult, if not impossible, due to inherent network delays encountered with propagation, protocol, and routing choice [34].

Fully automated robotics relieves the tedium of single-joint motions and allows the system to run unattended. It allows the operator to interact at a high level with the system and, therefore, is a good candidate for long distance interactions. However, full automation can require significant programming time and is often not practical for research or diagnostic operations.

The term 'telerobotics' [35] is derived from the merging of teleoperation (intensive human interaction moving one joint at time) and fully automated robotics (repetitive motions without any human interactions). It allows the operator to define the level between full automation and teleoperation on an operation by operation basis. A short teleoperated operation might be a test of the Z-axis to ensure engagement of the surface or perhaps the taking of a single scan line of data. After such an initial check, the operator could define a full image to be taken under robotic control. The tradeoff between the two modes of interaction depends on the desired task at hand and the distances involved.

\subsubsection{Control and Image Standards}

Telerobotics requires the development of standard interfaces between the controller and the user program. This takes the form of both control interfaces for the physical motion and image standards for feedback to the operator and analysis of the scanned surface. A meeting was convened in conjunction with the $4^{\text {th }}$ Workshop on Industrial Applications of Scanned Probe Microscopy held at NIST in May, 1997, to explore these standards issues.

The workshop was attended by representatives from the major SPM vendors at the time and several NIST staff members. The NAMT Nanomanufacturing project was first presented to give the telerobotic project task some context for the vendors. Detailed discussions centered on the issues of remote control, diagnostics, and image standardization. A lively discussion was held on the pros and cons of remote operation. In general, while vendors acknowledged the usefulness of remote diagnostics, many were uncomfortable with the idea of remote operation stating safety and sample preparation concerns. The discussion of a potential image standard garnered more support for simple X-Y scans, but stalled when the discussion turned to more complex images and techniques such as force curves. The general feeling was that the industry was still in a formative stage and not ready for an image standard that could cover all current and future image possibilities.

\subsubsection{Remote Diagnostics Testbed}

Another task was the development of a testbed to demonstrate remote diagnostic operation of a SPM and to identify standard interface needs. Based on the feedback of the workshop, this work focused on only high-level diagnostic operations and not complete low-level remote operations. 
The effort was carried out in collaboration with the University of North Carolina at Chapel Hill. Researchers there had worked closely with TopoMetrix Inc. [36] to define a low-level interface into the control system of the TopoMetrix SPM. This interface allowed the researchers to set gains, define simple trajectories for the microscope to perform, and in general, control the microscope in the same manner as an operator at the console. Real-time access to the control system and the image allowed a closed loop system called the Nanomanipulator [37,38] to be developed.

The Nanomanipulator system is capable of both low-level operations, such as moving a component along a single axis, as well as higher-level operations such as scanning images. The system is coupled with a high-speed graphic display and a haptic device to let the researcher 'feel' the surface under study. It has proven to be an excellent testbed for determining the tradeoffs involved between user access, network delays, and appropriate level of telerobotic operation for remote operation of SPMs.

We performed experiments on the Nanomanipulator system using a number of different control and network interfaces. The networks under consideration used either the industry standard transmission control protocol / internet protocol (TCP/IP) or the emerging Asynchronous Transfer Mode (ATM) protocol. ATM was developed to deliver data packets in a deterministic manner, which is often needed for the demands of networked multimedia data. TCP/IP ensures delivery of all data packets, but in a non-deterministic manner. These experiments confirmed the importance of a smooth, uninterrupted data stream to and from the SPM when performing lowlevel operations or very high-speed image transfers. This was achieved by TCP/IP over short distances on a lightly loaded network, or by ATM under most conditions.

\subsubsection{Recommendations}

The time is right for the SPM community to develop image and high-level control standards. The laboratory systems developed in the early and mid 1990s have given rise to the production systems of today. As the SPM finds its way out of the individual researcher's lab and onto the factory floor, the need for control and image standards increases. An image standard will not only allow free interchange of data between researchers; it will also allow third party vendors to supply image analysis packages and other add-on packages for inclusion on the factory floor. Although true remote operation may only make sense for laboratory experimentation, remote diagnostics will rise in importance as more SPMs are placed in factory settings. 


\section{Conclusions and Future Work}

In the atom-based standards field, we will continue to develop $\operatorname{Si}(111)$ specimens as single-atom step height standards. The work to determine the average step height is completed, and the manufacture of these specimens is practical. The specimens are now commercially available. In future projects, we will pursue standardization work to develop a user friendly measurement procedure that employs the standard to calibrate profiling instruments operating at the highest levels of resolution.

We will also continue to work on the critical problem of fabricating counted atom linewidth standards. This will involve developing a procedure for fabrication of clearly defined linewidth structures, then cleaning and annealing them to produce crystalline surfaces whose atoms can be measured and counted without degrading or melting their overall linewidth shapes in the process.

The use of mobile UHV environments may be further off. On one hand, UHV systems could become crucial for semiconductor manufacturing as functional thin films such as gate oxides approach atomic size thicknesses. Surface contamination before or after the fabrication of such thin layers could change their properties significantly. In addition, the need for delicate standards that must remain in UHV their entire lives may become greater if there is no other way to maintain highly perfect nanometer size dimensional standards. On the other hand, it may not be necessary to handle an artifact entirely in UHV. Other types of controlled environments may be acceptable, such as high vacuum, rough vacuum, clean gas, or clean atmospheric conditions. Atmospheric clean environments may suffice for housing many types of devices and standards, or centralized systems with all the UHV fabrication tools integrated into them may preclude the need for sample handoff between manipulators. Even so, the UHV community in general may profit greatly by developing standardized sample holders and manipulators that allow handoff of samples between them.

If there is a long-range need for distributed UHV systems allowing the atom-based dimensional artifacts to be used by many different users, arriving at some standards for both the transport container interfaces and the internal mechanisms will be necessary. This work will need to be closely coordinated with industrial partners such as vacuum equipment suppliers and end users of artifacts.

\section{Acknowledgments}

The authors are grateful to C. Jensen for designs and fabrication of a number of elements of the ATS, to E. Williams, V.W. Tsai, and Z. Hui at the University of Maryland for fabrication of the Si step height specimens and other technical contributions, to R. Fejol for work on the development of the design repository for the ATS and its elements, to N.G. Orji and R. Dixson for analysis of the round robin data for the $\mathrm{Si}(111)$ single-atom step height specimens, to $\mathrm{B}$. Young for creation of the Vacuum Suitcase videotape and website, to Michael Lin and Alice Ling for their assistance in developing the ATS data logger, and to V.S. Gagne for preparation of the text and graphics. The work was supported in part by the MEL NAMT Program and in part by the NIST Office of Microelectronics Programs. 


\section{References}

Note: The references shown in bold are publications of the Nanomanufacturing of AtomBased Dimensional Standards project.

[1] Bello, M.A.; Densock, R.J.; Swyt, D.A.; Land, J.L.; Stieren, D.C.; Wavering, A.J.; Donmez, M.A.; Christopher, N.B.; Carver, G.P., NIST: Toward 21st Century

Information-Based Manufacturing, NIST Special Publication 913, Gaithersburg, MD, September 1997.

[2] Stieren, D.C.; Densock, R.J.; Luce, M.E., National Advanced Manufacturing Testbed, NISTIR 6383 (National Institute of Standards and Technology, Gaithersburg, MD; August 1999). 25 pp.

[3] Stieren, D.C.; Luce, M.E., Information-Based Manufacturing Program Summary and Accomplishments, NISTIR 6490 (National Institute of Standards and Technology, Gaithersburg, MD; March 2000). 52 pp.

[4] International Technology Roadmap for Semiconductors: 1999 (Semiconductor Industry Association, Austin, TX, 1999).

[5] Vezzetti, C.F.; Varner, R.N.; Potzick, J.E., Antireflecting-Chromium Linewidth Standard, SRM 473, for Calibration of Optical Microscope Linewidth Measuring Systems, NIST Special Publication 260-119 (National Institute of Standards and Technology, Gaithersburg, MD; September 1992). 36 pp.

[6 ] Granneman, E.H.A., Trends in Contamination Control in IC Production Tools, Solid State Technology 40(7):225, July 1997.

[7] Parikh, M.; Kaempf, U., SMIF - A Technology for Wafer Cassette Transfer in VLSI Manufacturing, Solid State Technology 27 (7): 111-115; 1984.

[8] Stulen, R.H.; Sweeney, D.W., Extreme Ultraviolet Lithography, IEEE J. Quantum Elect. 35: 694-699; 1999.

[9] Hawryluk, A.M., Characterization and Possible Repair of Defects in SXPL Masks, OSA Proc. on Soft X-Ray Projection Lithography 18: 43-46; 1993.

[10] Singer, P.E., Diagnostics: Monitoring Tool Performance, Semiconductor International $\underline{24}$ (3): $27 ; 2001$. 
[11] See, for example, Tsai, V.W.; Wang, X.-S.; Williams, E.D.; Schneir, J.; Dixson, R.D., Conformal Oxides on Silicon Surfaces, Appl. Phys. Lett. 71 (11): 1495; 1997, and references contained therein.

[12] See: Mohr, P.J.; Taylor, B.N., CODATA Recommended Values of the Fundamental Physical Constants: 1998, Journal of Physical and Chemical Reference Data 28 (6); 1999; and Reviews of Modern Physics 72 (2): 351-495; 2000.

[13] Tsai, V.W.; Vorburger, T.V.; Dixson, R.; Fu, J.; Köning, R.; Silver, R.; Williams, E.D., The Study of Silicon Stepped Surfaces as Atomic Force Microscope Calibration Standards with a Calibrated AFM at NIST, in Characterization and Metrology for ULSI Technology: 1998 International Conference, D.G. Seiler, et al., eds. (AIP Press, 1998), pp. 839-842.

[14] Dixson, R.; Orji, N.G.; Fu, J.; Tsai, V.W.; Williams, E.D.; Kacker, R.; Vorburger, T.V.; Edwards, H.; Cook, D.; West, P.; Nyffenegger, R., Silicon Single Atom Steps as AFM Height Standards, Proc. SPIE 4344: 157-168; 2001.

[15] Phaneuf, R.J.; Williams, E.D., Surface Phase Separation of Vicinal Si (111), Phys. Rev. Lett. 58: 2563; 1987.

[16] Noh, D.Y.; Blum, K.I.; Ramstad, M.J.;. Birgeneau, R.J., Long-range Coherence of Macroscopic Phase Separation of Steps on Vicinal Si(111), Phys. Rev. B 44: 10969; 1991.

[17] Suzuki, M.; Aoyama, S.; Futatsuki, T.; Kelly, A.J.; Osada, T.; Nakano, A.; Sakasibara, Y.; Suzuki, Y.; Takami, H.; Takenobu, I.; Yasutake, M., Standardized Procedure for Calibrating Height Scales in Atomic Force Microscopy on the Order of $1 \mathrm{~nm}, J$. Vac.

Sci. Technol. A 14 (3): 1228-1232; 1996.

[18] UC Standard, One-Nanometer-Order Z-Axis Calibration of AFM (Ultraclean Society, Tokyo, 1995).

[19] Fu, J.; Tsai, V.W.; Köning, R.; Dixson, R.; Vorburger, T.V., Algorithms for Calculating Single Atom Step Heights, Nanotechnology 10: 428-433; 1999.

[20] Photomask Linewidth Standards, Micrometrology Section, National Physical Laboratory Queens Road, Teddington, Middlesex, TW11 0LW, England.

[21] Silver, R.M.; Jensen, C.P.; Tsai, V.; Fu, J.; Villarrubia, J.; Teague, E.C., Developing a Method to Determine Linewidth Based on Counting the AtomSpacings Across a Line, Proc. SPIE 3332: 441-460; 1998. 
[22] Villarrubia, J.S.; Dixson, R.; Jones, S.; Lowney, J.R.; Postek, M.R.; Allen, R.A.; Cresswell, M.W., Intercomparison of SEM, AFM, and Electrical Linewidths, Proc. SPIE 3677: 587-598; 1999.

[23] Müller, E.W., Field-Ion Microscopy and the Electronic Structure of Metal Surfaces, Quarterly Reviews XXIII (2) (The Chemical Society, London, 1969), pp 177-186.

[24] Fink, H.W., Mono-atomic Tips for Scanning Tunneling Microscopy, IBM J. Res Develop. 30 (5): 460-465; 1986.

[25] Rao, P.V.M.; Jensen, C.P.; and Silver, R.M., A Generic Model for STM Tip Geometry Measured with FIM (submitted to Ultramicroscopy).

[26] Hobson, J.P.; Kornelsen, E.V., UHV Technique for Intervacuum Sample Transfer, J. Vac. Sci. Technol. 16 (2): 701-710; 1979.

[27] Young, B., Vacuum Suitcase, http://www.mel.nist.gov/gallery/vcase/vscase1.htm, Last updated June 8, 2001.

[28] Korczynski, E., Design Challenges in Vacuum Robotics, Solid State Technology, October 1996.

[29] Nidamarthi, S.; Allen, R.H.; Sriram, R.D., Observations from Supplementing the Traditional Design Process via Internet-based Collaboration Tools, International Journal of Computer-Integrated Manufacturing 14 (1): 95-107; 2001.

[30] Allen, R. H.; Fijol, R. J.; Szykman, S.; Sriram, R.D., Representing an Artifact System in a Design Repository: A Case Study, Proc. DETC'00/CIE, D. E. Lee, ed. (American Society of Mechanical Engineers, New York, September 2000); Paper No. CIE 14608, 8 pp.

[31] Allen, R.H.; Nidamarthi, S.; Regalla, S.P.; Sriram, R.D., Enhancing Collaboration Using an Internet-Integrated Workbench, Proc. DETC'99, M. Gartner et al., eds. (American Society of Mechanical Engineers, New York, September 1999); Paper No. DAC 8573, 12 pp.

[32] Nidamarthi, S.; Allen, R.H.; Prakash, S.R.; Sriram, R.D., Observations from Multidisciplinary, Internet-based Collaboration on a Practical Design Project, International Conference on Engineering Design, ICED99 2.: 709-714; August 1999.

[33] Allen, R.H.; Nidamarthi, S.; Rao, P.V.M.; Rhorer, R.; Sriram, R.D.; Teague, E.C., Collaborating on the Design and Manufacture of an Artifact Transport System: A Case Study in VRML as a Visualization Tool for Consensus Building, Proc. DETC'98, M. Gartner et al., eds. (American Society of Mechanical Engineers, New York, September 1998, Paper No. DAC 5600, 12 pp. 
[34] Clark, M.; Jeffray, K., Application-Level Measurements of Performance on the vBNS, IEEE Intl. Conference on Multimedia Computing and Systems, June 1999, Florence, Italy.

[35] Lumia, R.; Michaloski, J.; Russell, R.; Wheatley, T.; Backe, P.; Lee, S.; Steele, R.; Unified Telerobotic Architecture Project (UTAP) Standard Interface Environment (SIE), NISTIR 5658; National Institute of Standards and Technology, Gaithersburg, MD, May 1995.

[36] TopoMetrix has merged with Park Scientific and is now called TM Microscopes.

[37] Taylor, R.M., The Nanomanipulator: A Virtual-Reality Interface to a Scanning Tunneling Microscope, Ph.D. Dissertation, University of North Carolina, Chapel Hill, TR94-030, May 1994.

[38] Falvo, M.; Superfine, R.; Washburn, S.; Finch, M.; Taylor, R.M.; Chi, V.L.; Brooks Jr., F.P., The Nanomanipulator: A Teleoperator for Manipulating Materials at the Nanometer Scale, Proceedings of the International Symposium on the Science and Technology of Atomically Engineered Materials, (Richmond, VA, Oct 30 - Nov 4, 1995). World Scientific Publishing 1996. pp. 579-586. 